\title{
The Amblyomma maculatum Koch, 1844 (Acari: Ixodidae) group of ticks: phenotypic plasticity or incipient speciation?
}

Paula Lado ${ }^{1,2^{*}}$, Santiago Nava ${ }^{3}$, Leonardo Mendoza-Uribe ${ }^{4}$, Abraham G. Caceres5 ${ }^{5,6}$, Jesus Delgado-de la Mora ${ }^{7}$, Jesus D. Licona-Enriquez ${ }^{7}$, David Delgado-de la Mora ${ }^{8}$, Marcelo B. Labruna ${ }^{9}$, Lance A. Durden ${ }^{10}$, Michelle E. J. Allerdice ${ }^{11}$, Christopher D. Paddock ${ }^{11}$, Matias P. J. Szabó ${ }^{12}$, José M. Venzal ${ }^{13}$,

Alberto A. Guglielmone ${ }^{3}$ and Lorenza Beati ${ }^{1}$

\begin{abstract}
Background: The goal of this study was to reassess the taxonomic status of A. maculatum, A. triste and A. tigrinum by phylogenetic analysis of five molecular markers [four mitochondrial: $125 \mathrm{SDNA}$, $16 \mathrm{~S}$ rDNA, the control region (DL) and cytochrome c oxidase 1 (cox1), and one nuclear: ribosomal intergenic transcribed spacer 2 (ITS2)]. In addition, the phenotypic diversity of adult ticks identified as A. maculatum and A. triste from geographically distinct populations was thoroughly re-examined.

Results: Microscopic examination identified four putative morphotypes distinguishable by disjunct geographical ranges, but very scant fixed characters. Analysis of the separated mitochondrial datasets mostly resulted in conflicting tree topologies. Nuclear gene sequences were almost identical throughout the geographical ranges of the two species, suggesting a very recent, almost explosive radiation of the terminal operational taxonomic units. Analysis of concatenated molecular datasets was more informative and indicated that, although genetically very close to the A. maculatum - A. triste lineage, A. tigrinum was a monophyletic separate entity. Within the A. maculatum - A. triste cluster, three main clades were supported. The two morphotypes, corresponding to the western North American and eastern North American populations, consistently grouped in a single monophyletic clade with many shared mitochondrial sequences among ticks of the two areas. Ticks from the two remaining morphotypes, south-eastern South America and Peruvian, corresponded to two distinct clades.

Conclusions: Given the paucity of morphological characters, the minimal genetic distance separating morphotypes, and more importantly the fact that two morphotypes are genetically indistinguishable, our data suggest that $A$. maculatum and A. triste should be synonymized and that morphological differences merely reflect very recent local adaptation to distinct environments in taxa that might be undergoing the first steps of speciation but have yet to complete lineage sorting. Nonetheless, future investigations using more sensitive nuclear markers and/or crossbreeding experiments might reveal the occurrence of very rapid speciation events in this group of taxa. Tentative node dating revealed that the $A$. tigrinum and A. maculatum - A. triste clades split about 2 Mya, while the A. maculatum - A.triste cluster radiated no earlier than 700,000 years ago.
\end{abstract}

Keywords: Amblyomma, Systematics, Ticks, Amblyomma maculatum, Taxonomic reassessment, Phylogenetic analysis, Amblyomma triste

\footnotetext{
* Correspondence: ladohenaise.1@osu.edu

'U. S. National Tick Collection, Institute for Coastal Plain Science, Georgia

Southern University, Statesboro, GA 30460, USA

${ }^{2}$ Present address: Department of Evolution, Ecology, and Organismal Biology,

The Ohio State University, Columbus, OH 43212, USA

Full list of author information is available at the end of the article
}

(c) The Author(s). 2018 Open Access This article is distributed under the terms of the Creative Commons Attribution 4.0 International License (http://creativecommons.org/licenses/by/4.0/), which permits unrestricted use, distribution, and reproduction in any medium, provided you give appropriate credit to the original author(s) and the source, provide a link to the Creative Commons license, and indicate if changes were made. The Creative Commons Public Domain Dedication waiver (http://creativecommons.org/publicdomain/zero/1.0/) applies to the data made available in this article, unless otherwise stated. 


\section{Background}

The Amblyomma maculatum group includes, according to Camicas et al. [1], the following species: A. maculatum Koch, 1844; Amblyomma neumanni Ribaga, 1902; Amblyomma parvitarsum Neumann, 1901; Amblyomma tigrinum Koch, 1844 and Amblyomma triste Koch, 1844. Together with the Amblyomma ovale Koch, 1844 group which encompasses A. ovale and Amblyomma aureolatum (Pallas, 1772), they have been clustered by Camicas et al. [1] in a revised version of subgenus Anastosiella, originally erected by Santos Dias [2], who also included within this subgenus Amblyomma pecarium Dunn, 1933 and Amblyomma brasiliense Aragão, 1908. However, within the $A$. maculatum group, the adult and immature stages of $A$. neumanni and $A$. parvitarsum are morphologically distinguishable from the other species. Unlike the other three taxa, they are both characterized by incomplete marginal grooves in males, with $A$. parvitarsum having beady and orbited eyes [3]. In females, aside from A. neumanni, all species are glabrous dorsally. Amblyomma parvitarsum females also have beady and orbited eyes. Other diagnostic differences are listed in Estrada- Peña et al. [3], who suggested that $A$. neumanni and $A$. parvitarsum should be grouped with the $A$. ovale group in a yet to be determined subgenus, with $A$. maculatum, $A$. triste and A. tigrinum the only remaining members of the subgenus Anastosiella.

Members of the subgenus Anastosiella are morphologically very similar. This similarity is more apparent between A. maculatum and A. triste, and is examined in depth in this study. Koch [4] briefly described the three taxa based on males of $A$. maculatum and A. tigrinum, and a female of $A$. triste. He completed his description in 1850 [5] and essentially reported differences in punctation and ornamentation. Neumann [6] synonymyzed $A$. tigrinum and $A$. triste with $A$. maculatum after failing to observe differences in the shape of distal spines (modified setae) on tibiae II to IV (called tarsi by [6], protarsi by [7] and metatarsi by [8]). Kohls [8] reestablished $A$. tigrinum and $A$. triste as valid species and completely redescribed the three taxa. In that work, the characters differentiating the three species were the presence/absence of tubercles on the festoons, and the presence of one or two spurs on "metatarsi" of legs II-IV. Nevertheless, the identification chiefly of A. maculatum and $A$. triste has consistently been challenging [3, 9-11] and marred by frequent misidentifications [12-14]. Taxonomic issues are not limited to adult stages: immatures, for which taxonomic keys are available, are even more difficult to differentiate $[3,11,15-17]$ with, again, the exception of $A$. parvitarsum and A. neumanni which are easily separated from the other three species.

The distribution of A. maculatum is presumably confined to the southern USA, Central America and some areas of Colombia, Venezuela, Peru and Ecuador, whereas
A. tigrinum is reported to occur only in South American countries [8, 18-21]. Amblyomma triste was regarded as being exclusively South American in its distribution until recently, when it was reported from Mexico and the southwestern USA $[11,22]$, thus increasing the number of tick species with both, a Neotropical and Nearctic distribution [9].

A thorough review of the taxonomic status of this complex of species is essential, not only for systematic reasons, but also because $A$. maculatum, $A$. triste and $A$. tigrinum are recognized increasingly as vectors of pathogens of veterinary and public health importance throughout the Americas, particularly Rickettsia parkeri [23-34] and Hepatozoon americanum [35].

Molecular techniques used to infer phylogenetic relationships and evaluate the taxonomic status of the different species of the $A$. maculatum group have yet to be applied in a comprehensive manner. Preliminary reports based on the analysis of $16 S \mathrm{rDNA}$ sequences confirmed that A. maculatum, $A$. triste and $A$. tigrinum are closely related to each other, whereas $A$. neumanni and $A$. parvitarsum are distinct not only from each other and from the rest of the $A$. maculatum group of taxa, but do not cluster with the $A$. ovale group of species [3].

The aim of this study was to reassess the taxonomic status of $A$. maculatum, $A$. triste and $A$. tigrinum by analyzing their phylogenetic relationships determined by comparisons of one nuclear and four mitochondrial gene sequences. In addition, a comprehensive morphological analysis of the adult stage of $A$. maculatum and $A$. triste is presented. Our working hypothesis is that $A$. maculatum, $A$. triste and A. tigrinum comprise three separate species.

\section{Results \\ Morphological reassessment of the A. maculatum - $A$. triste specimens}

Microscopic examination identified four morphological groups that, for the sake of simplicity, will be called here Morphotypes I, II, III and IV. The four morphotypes have disjunct geographical distributions (Fig. 1; morphotype distribution for the samples used in the molecular analyses) and feature many common and a few distinctive character combinations (Table 1). The shape of modified setae on the ventral distal end of the tibiae of legs II-IV (tibial armature) have been commonly used to differentiate ticks of the $A$. maculatum group. They have been called spines, setae or spurs in earlier literature. In the following descriptions we will use the term "seta" if the structure is fine and weakly sclerotized, "spine" if the structure is thick and heavily sclerotized. For measurements, refer to scales on the images. 


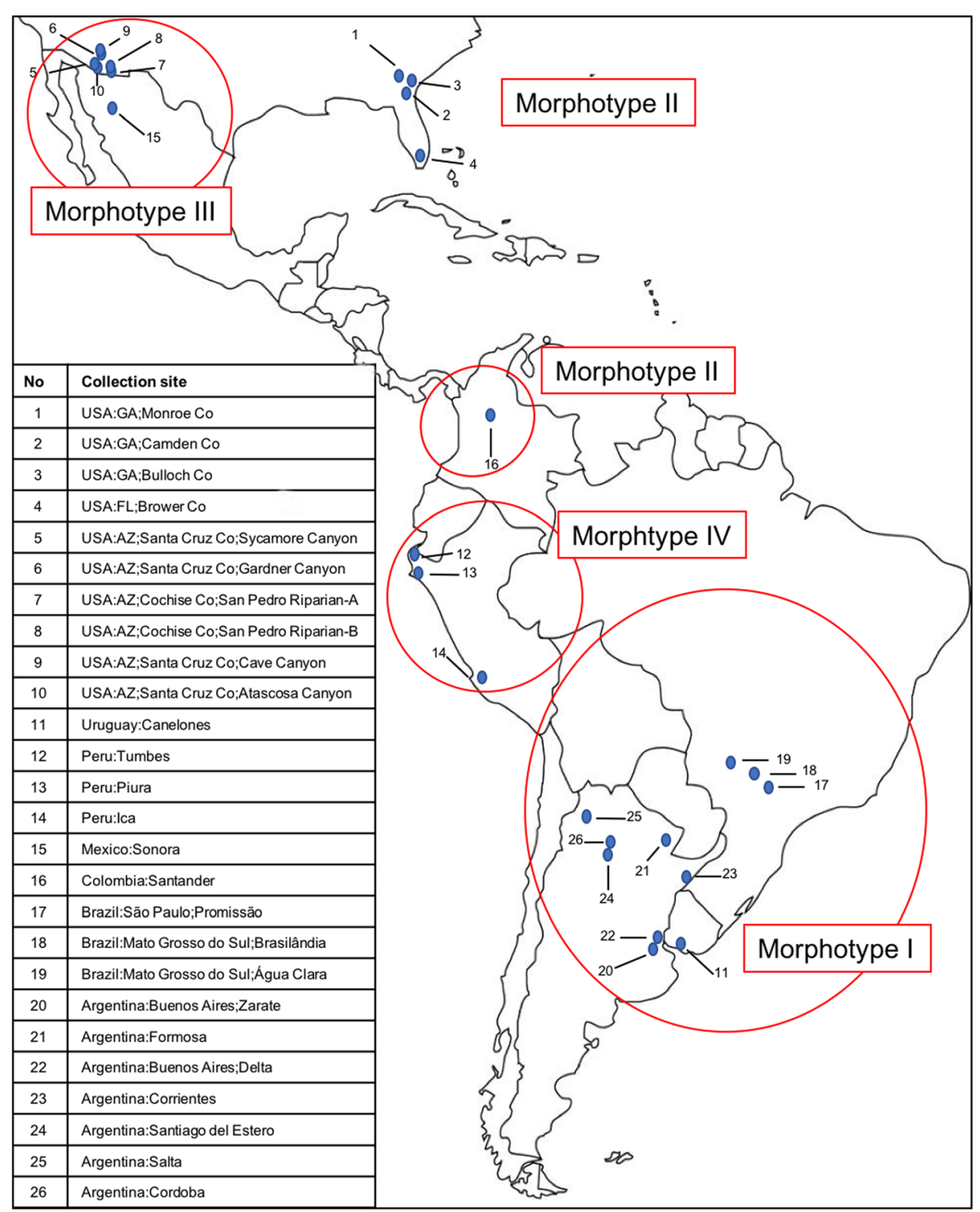

Fig. 1 Map showing the geographical locations of ticks used for molecular analysis in this study and their corresponding morphotypes

\section{Common characters in males (Figs. 2, 3, 5, 6)}

Body outline elongate-oval, narrower anterior to eyes; scapulae rounded; eyes flat. Scutum ornate, with reddish brown spots outlined by pale yellowish enameled stripes; postero-median spot extending anteriorly to level of spiracular plates; postero-accessory spots parallel to postero-median spot; three lateral spots large and sometimes fused; cervical spots narrow, first diverging, then slightly converging posteriorly; central area long, extending to mid-length of scutum. Chitinous scutes sometimes reduced to a tubercle, but always present on ventral surface of festoons. Basis capituli dorsally sub-rectangular, cornua long. Hypostome spatulate, dental formula 3:3. Legs: trochanters without spurs, coxa I with two distinct spurs, external spur long and sharp, internal spur as a small tubercle; coxae II-III with triangular, short, blunt spur each; coxa IV with narrow long sharp spur.

\section{Common characters in females (Figs. 2-6)}

Body outline oval. Scapulae rounded, cervical grooves deep anteriorly, shallow posteriorly, sigmoid in shape; eyes flat. Small chitinous tubercles dorsally visible at postero-medial edge of festoons. Scutum ornate, extensively pale yellowish, cervical spots narrow and divergent posteriorly, brown central area long, narrow. Notum glabrous dorsally, but with fine, very short, evenly distributed setae ventrally. Basis capituli dorsally sub-rectangular, cornua absent, porose areas oval. Hypostome spatulate, dental formula 3:3. Legs: coxa I with two distinct spurs, 
Table 1 Tick samples used for the molecular portion of this study and the gene amplifications results. Crosses indicate successful amplification and sequencing of the corresponding molecular marker

\begin{tabular}{|c|c|c|c|c|c|c|c|c|c|c|c|c|c|}
\hline Species & Mt & Voucher ID & Country & $\begin{array}{l}\text { State/ province/ } \\
\text { department }\end{array}$ & Locality & Code & Coordinates & 125 & $16 S$ & $\mathrm{DL}$ & ITS2 & $\operatorname{cox} 1$ & $\cos 2$ \\
\hline A. neumanni & na & & Argentina & Cordoba & Dean Funes & AN & $\begin{array}{l}30^{\circ} 26^{\prime} \mathrm{S} \\
64^{\circ} 20^{\prime} \mathrm{W}\end{array}$ & $\times$ & $\times$ & $\times$ & $\times$ & $x$ & $\times$ \\
\hline A. parvitarsum & na & & Argentina & Salta & $\begin{array}{l}45 \mathrm{~km} \text { E of } \mathrm{S} \text {. } \\
\text { Antonio de los } \\
\text { Cobres }\end{array}$ & AP & $\begin{array}{l}24^{\circ} 22^{\prime} \mathrm{S} \\
66^{\circ} 42^{\prime} \mathrm{W}\end{array}$ & $x$ & $\times$ & & $x$ & $x$ & $x$ \\
\hline A. tigrinum & na & & Argentina & SDE & Pozo Hondo & SDE_16A & $\begin{array}{l}27^{\circ} 10^{\prime} \mathrm{S} \\
64^{\circ} 30^{\prime} \mathrm{W}\end{array}$ & $x$ & $\times$ & & $\times$ & $x$ & $x$ \\
\hline A. tigrinum & na & & Argentina & SDE & Pozo Hondo & SDE_16B & $\begin{array}{l}27^{\circ} 10^{\prime} \mathrm{S} \\
64^{\circ} 30^{\prime} \mathrm{W}\end{array}$ & $\times$ & $\times$ & $\times$ & $\times$ & $x$ & \\
\hline A. triste & I & & Argentina & Corrientes & Colonia Pellegrini & $C R \_15 A$ & $\begin{array}{l}29^{\circ} 00^{\prime} \mathrm{S} \\
57^{\circ} 30^{\prime} \mathrm{W}\end{array}$ & $x$ & $x$ & $\times$ & $x$ & $\times$ & \\
\hline A. triste & I & INTA 1979 & Argentina & $\mathrm{BA}$ & Inta Delta & BA_AT3A & $\begin{array}{l}34^{\circ} 11^{\prime} \mathrm{S} \\
58^{\circ} 50^{\prime} \mathrm{W}\end{array}$ & $x$ & & & & $x$ & \\
\hline A. triste & I & INTA 1980 & Argentina & $\mathrm{BA}$ & Inta Delta & BA_AT3B & $\begin{array}{l}34^{\circ} 11^{\prime} \mathrm{S} \\
58^{\circ} 50^{\prime} \mathrm{W}\end{array}$ & & & & & $x$ & \\
\hline A. triste & I & INTA 1981 & Argentina & $\mathrm{BA}$ & Inta Delta & BA_AT3C & $\begin{array}{l}34^{\circ} 11^{\prime} \mathrm{S} \\
58^{\circ} 50^{\prime} \mathrm{W}\end{array}$ & $x$ & & & & $x$ & \\
\hline A. triste & 1 & INTA 1982 & Argentina & $\mathrm{BA}$ & Inta Delta & BA_AT3D & $\begin{array}{l}34^{\circ} 11^{\prime} \mathrm{S} \\
58^{\circ} 50^{\prime} \mathrm{W}\end{array}$ & & & & & $x$ & $x$ \\
\hline A. triste & I & INTA 1983 & Argentina & $\mathrm{BA}$ & Inta Delta & BA_AT3E & $\begin{array}{l}34^{\circ} 11^{\prime} \mathrm{S} \\
58^{\circ} 50^{\prime} \mathrm{W}\end{array}$ & & & $x$ & & $x$ & \\
\hline A. triste & I & INTA 1984 & Argentina & $\mathrm{BA}$ & Inta Delta & BA_1A & $\begin{array}{l}34^{\circ} 11^{\prime} \mathrm{S} \\
58^{\circ} 50^{\prime} \mathrm{W}\end{array}$ & $x$ & $\times$ & $x$ & $x$ & $x$ & \\
\hline A. triste & I & INTA 1985 & Argentina & BA & Inta Delta & BA_1A2 & $\begin{array}{l}34^{\circ} 11^{\prime} \mathrm{S} \\
58^{\circ} 50^{\prime} \mathrm{W}\end{array}$ & $x$ & $x$ & $x$ & & $x$ & \\
\hline A. triste & I & INTA 1986 & Argentina & $\mathrm{BA}$ & Inta Delta & BA_1B & $\begin{array}{l}34^{\circ} 11^{\prime} \mathrm{S} \\
58^{\circ} 50^{\prime} \mathrm{W}\end{array}$ & $\times$ & $x$ & $x$ & $x$ & $x$ & \\
\hline A. triste & I & INTA 1987 & Argentina & $\mathrm{BA}$ & Inta Delta & BA_1C & $\begin{array}{l}34^{\circ} 11^{\prime} \mathrm{S} \\
58^{\circ} 50^{\prime} \mathrm{W}\end{array}$ & $x$ & $x$ & $\times$ & $x$ & $x$ & \\
\hline A. triste & I & INTA 1988 & Argentina & BA & Inta Delta & BA_1D & $\begin{array}{l}34^{\circ} 11^{\prime} \mathrm{S} \\
58^{\circ} 50^{\prime} \mathrm{W}\end{array}$ & & $x$ & & $x$ & $\times$ & \\
\hline A. triste & I & INTA 1989 & Argentina & $\mathrm{BA}$ & Inta Delta & BA_1E & $\begin{array}{l}34^{\circ} 11^{\prime} \mathrm{S} \\
58^{\circ} 50^{\prime} \mathrm{W}\end{array}$ & & $x$ & & $x$ & $x$ & \\
\hline A. triste & I & INTA 1990 & Argentina & $\mathrm{BA}$ & Inta Delta & BA_18A & $\begin{array}{l}34^{\circ} 11^{\prime} \mathrm{S} \\
58^{\circ} 50^{\prime} \mathrm{W}\end{array}$ & $\times$ & $x$ & $\times$ & & $x$ & \\
\hline A. triste & I & INTA 1991 & Argentina & BA & Inta Delta & BA_18B & $\begin{array}{l}34^{\circ} 11^{\prime} \mathrm{S}, \\
58^{\circ} 50^{\prime} \mathrm{W}\end{array}$ & $x$ & $\times$ & $\times$ & $x$ & & \\
\hline A. triste & I & INTA 1992 & Argentina & BA & Inta Delta & BA_18C & $\begin{array}{l}34^{\circ} 11^{\prime} \mathrm{S} \\
58^{\circ} 50^{\prime} \mathrm{W}\end{array}$ & $\times$ & $\times$ & $\times$ & $x$ & & \\
\hline A. triste & I & INTA 2096 & Argentina & Formosa & Reserva El Bagual & FO_12A & $\begin{array}{l}26^{\circ} 10^{\prime} \mathrm{S} \\
58^{\circ} 56^{\prime} \mathrm{W}\end{array}$ & $\times$ & $x$ & $\times$ & $x$ & $x$ & $x$ \\
\hline A. triste & I & INTA 2097 & Argentina & Formosa & Reserva El Bagual & FO_12B & $\begin{array}{l}26^{\circ} 10^{\prime} \mathrm{S} \\
58^{\circ} 56^{\prime} \mathrm{W}\end{array}$ & & $x$ & $\times$ & & $x$ & \\
\hline A. triste & I & INTA 2098 & Argentina & Formosa & Reserva El Bagual & FO_12C & $\begin{array}{l}26^{\circ} 10^{\prime} \mathrm{S} \\
58^{\circ} 56^{\prime} \mathrm{W}\end{array}$ & & $x$ & $\times$ & $x$ & & \\
\hline A. triste & I & INTA 2099 & Argentina & Formosa & Reserva El Bagual & FO_12D & $\begin{array}{l}26^{\circ} 10^{\prime} \mathrm{S} \\
58^{\circ} 56^{\prime} \mathrm{W}\end{array}$ & $x$ & $x$ & $\times$ & $x$ & & \\
\hline A. triste & I & INTA 2100 & Argentina & Formosa & Reserva El Bagual & FO_12E & $\begin{array}{l}26^{\circ} 10^{\prime} \mathrm{S} \\
58^{\circ} 56^{\prime} \mathrm{W}\end{array}$ & $x$ & $\times$ & $\times$ & $x$ & & \\
\hline A. triste & I & & Argentina & BA & Zarate & BA_AT1A & $\begin{array}{l}34^{\circ} 05^{\prime} \mathrm{S} \\
59^{\circ} 00^{\prime} \mathrm{W}\end{array}$ & & & & $x$ & $x$ & \\
\hline
\end{tabular}


Table 1 Tick samples used for the molecular portion of this study and the gene amplifications results. Crosses indicate successful amplification and sequencing of the corresponding molecular marker (Continued)

\begin{tabular}{|c|c|c|c|c|c|c|c|c|c|c|c|c|c|}
\hline Species & Mt & Voucher ID & Country & $\begin{array}{l}\text { State/ province/ } \\
\text { department }\end{array}$ & Locality & Code & Coordinates & 125 & 165 & $\mathrm{DL}$ & ITS2 & $\cos 1$ & $\cos 2$ \\
\hline A. triste & I & & Argentina & $B A$ & Zarate & BA_AT1B & $\begin{array}{l}34^{\circ} 05^{\prime} \mathrm{S} \\
59^{\circ} 00^{\prime} \mathrm{W}\end{array}$ & $\times$ & & & & $\times$ & \\
\hline A. triste & I & & Argentina & BA & Zarate & BA_AT1C & $\begin{array}{l}34^{\circ} 05^{\prime} \mathrm{S}, \\
59^{\circ} 00^{\prime} \mathrm{W}\end{array}$ & $x$ & & & & $\times$ & \\
\hline A. triste & I & & Argentina & BA & Zarate & BA_17A & $\begin{array}{l}34^{\circ} 05^{\prime} \mathrm{S} \\
59^{\circ} 00^{\prime} \mathrm{W}\end{array}$ & $\times$ & $\times$ & & $x$ & $\times$ & \\
\hline A. triste & 1 & & Argentina & BA & Zarate & BA_17B & $\begin{array}{l}34^{\circ} 05^{\prime} \mathrm{S} \\
59^{\circ} 00^{\prime} \mathrm{W}\end{array}$ & $\times$ & $\times$ & $x$ & $x$ & $\times$ & \\
\hline A. triste & 1 & & Argentina & BA & Zarate & BA_17C & $\begin{array}{l}34^{\circ} 05^{\prime} \mathrm{S} \\
59^{\circ} 00^{\prime} \mathrm{W}\end{array}$ & $\times$ & $\times$ & $x$ & $x$ & $\times$ & \\
\hline A. tigrinum & na & & Brazil & Goias & Caldas Novas & GO_11A & $\begin{array}{l}17^{\circ} 45^{\prime} \mathrm{S} \\
48^{\circ} 38^{\prime} \mathrm{W}\end{array}$ & $\times$ & $\times$ & $\times$ & & $\times$ & \\
\hline A. tigrinum & na & & Brazil & Goias & Mineiros & GO_5A & $\begin{array}{l}17^{\circ} 34^{\prime} \mathrm{S} \\
52^{\circ} 33^{\prime} \mathrm{W}\end{array}$ & $x$ & $\times$ & $x$ & & $\times$ & $\times$ \\
\hline A. tigrinum & na & & Brazil & Goias & Mineiros & GO_5A2 & $\begin{array}{l}17^{\circ} 34^{\prime} \mathrm{S} \\
52^{\circ} 33^{\prime} \mathrm{W}\end{array}$ & $\times$ & $\times$ & $\times$ & & $\times$ & \\
\hline A. tigrinum & na & & Brazil & Goias & Mineiros & GO_5B & $\begin{array}{l}17^{\circ} 34^{\prime} \mathrm{S} \\
52^{\circ} 33^{\prime} \mathrm{W}\end{array}$ & $\times$ & $\times$ & & & $\times$ & \\
\hline A. tigrinum & na & & Brazil & Goias & Mineiros & GO_5C & $\begin{array}{l}17^{\circ} 34^{\prime} \mathrm{S} \\
52^{\circ} 33^{\prime} \mathrm{W}\end{array}$ & $\times$ & $x$ & $\times$ & & $\times$ & $\times$ \\
\hline A. tigrinum & na & & Brazil & Goias & Mineiros & GO_5D & $\begin{array}{l}17^{\circ} 34^{\prime} \mathrm{S} \\
52^{\circ} 33^{\prime} \mathrm{W}\end{array}$ & & $x$ & $\times$ & & & \\
\hline A. tigrinum & na & & Brazil & Goias & Mineiros & GO_5E & $\begin{array}{l}17^{\circ} 34^{\prime} \mathrm{S} \\
52^{\circ} 33^{\prime} \mathrm{W}\end{array}$ & $\times$ & $x$ & $\times$ & & & \\
\hline A. tigrinum & na & & Brazil & Goias & Mineiros & GO_3A1 & $\begin{array}{l}17^{\circ} 34^{\prime} \mathrm{S} \\
52^{\circ} 33^{\prime} \mathrm{W}\end{array}$ & $\times$ & $x$ & $x$ & $x$ & $x$ & \\
\hline A. triste & 1 & USNMENT1430164 & Brazil & MGS & Agua Clara & MGS_6A & $\begin{array}{l}20^{\circ} 14^{\prime} \mathrm{S} \\
53^{\circ} 23^{\prime} \mathrm{W}\end{array}$ & $\times$ & $x$ & & $x$ & & \\
\hline A. triste & I & USNMENT1430165 & Brazil & MGS & Agua Clara & MGS_6A2 & $\begin{array}{l}20^{\circ} 14^{\prime} \mathrm{S} \\
53^{\circ} 23^{\prime} \mathrm{W}\end{array}$ & $\times$ & $x$ & $x$ & $x$ & $x$ & \\
\hline A. triste & 1 & USNMENT1430166 & Brazil & MGS & Agua Clara & MGS_6B & $\begin{array}{l}20^{\circ} 14^{\prime} S^{\prime} \\
53^{\circ} 23^{\prime} W\end{array}$ & $\times$ & $x$ & $x$ & $x$ & $\times$ & \\
\hline A. triste & I & USNMENT1430167 & Brazil & MGS & Agua Clara & MGS_6C & $\begin{array}{l}20^{\circ} 14^{\prime} \mathrm{S} \\
53^{\circ} 23^{\prime} \mathrm{W}\end{array}$ & $\times$ & $x$ & $x$ & $x$ & $\times$ & \\
\hline A. triste & I & USNMENT1430168 & Brazil & MGS & Agua Clara & MGS_6D & $\begin{array}{l}20^{\circ} 14^{\prime} \mathrm{S} \\
53^{\circ} 23^{\prime} \mathrm{W}\end{array}$ & $\times$ & $x$ & $x$ & $x$ & $x$ & \\
\hline A. triste & । & USNMENT1430169 & Brazil & MGS & Agua Clara & MGS_6E & $\begin{array}{l}20^{\circ} 14^{\prime} \mathrm{S} \\
53^{\circ} 23^{\prime} \mathrm{W}\end{array}$ & $\times$ & $x$ & $x$ & $x$ & & \\
\hline A. triste & I & USNMENT1430170 & Brazil & MGS & Agua Clara & MGS_6F & $\begin{array}{l}20^{\circ} 14^{\prime} \mathrm{S} \\
53^{\circ} 23^{\prime} \mathrm{W}\end{array}$ & & $x$ & $x$ & & $x$ & \\
\hline A. triste & 1 & USNMENT1430171 & Brazil & MGS & Agua Clara & MGS_6G & $\begin{array}{l}20^{\circ} 14^{\prime} \mathrm{S} \\
53^{\circ} 23^{\prime} \mathrm{W}\end{array}$ & & $x$ & $x$ & & $\times$ & \\
\hline A. triste & । & USNMENT1430172 & Brazil & MGS & Agua Clara & MGS_8A & $\begin{array}{l}20^{\circ} 14^{\prime} \mathrm{S} \\
53^{\circ} 23^{\prime} \mathrm{W}\end{array}$ & $x$ & $x$ & $x$ & $x$ & $\times$ & \\
\hline A. triste & I & USNMENT1430173 & Brazil & MGS & Agua Clara & MGS_8A2 & $\begin{array}{l}20^{\circ} 14^{\prime} \mathrm{S} \\
53^{\circ} 23^{\prime} \mathrm{W}\end{array}$ & $\times$ & $x$ & $x$ & $x$ & $\times$ & \\
\hline A. triste & I & USNMENT1430174 & Brazil & MGS & Agua Clara & MGS_8B & $\begin{array}{l}20^{\circ} 14^{\prime} \mathrm{S} \\
53^{\circ} 23^{\prime} \mathrm{W}\end{array}$ & $x$ & $x$ & $x$ & $x$ & $x$ & \\
\hline A. triste & I & USNMENT1430175 & Brazil & MGS & Agua Clara & MGS_8C & $\begin{array}{l}20^{\circ} 14^{\prime} \mathrm{S} \\
53^{\circ} 23^{\prime} \mathrm{W}\end{array}$ & $\times$ & $x$ & $\times$ & $x$ & & \\
\hline A. triste & I & USNMENT1430176 & Brazil & MGS & Agua Clara & MGS_8D & $\begin{array}{l}20^{\circ} 14^{\prime} \mathrm{S} \\
53^{\circ} 23^{\prime} \mathrm{W}\end{array}$ & & $x$ & & $x$ & $x$ & \\
\hline
\end{tabular}


Table 1 Tick samples used for the molecular portion of this study and the gene amplifications results. Crosses indicate successful amplification and sequencing of the corresponding molecular marker (Continued)

\begin{tabular}{|c|c|c|c|c|c|c|c|c|c|c|c|c|c|}
\hline Species & Mt & Voucher ID & Country & $\begin{array}{l}\text { State/ province/ } \\
\text { department }\end{array}$ & Locality & Code & Coordinates & 125 & 165 & $\mathrm{DL}$ & ITS2 & $\cos 1$ & $\cos 2$ \\
\hline A. triste & I & USNMENT1430177 & Brazil & MGS & Agua Clara & MGS_8E & $\begin{array}{l}20^{\circ} 14^{\prime} \mathrm{S} \\
53^{\circ} 23^{\prime} \mathrm{W}\end{array}$ & & $\times$ & $x$ & $x$ & & \\
\hline A. triste & I & USNMENT1430178 & Brazil & MGS & Agua Clara & MGS_8F & $\begin{array}{l}20^{\circ} 14^{\prime} \mathrm{S} \\
53^{\circ} 23^{\prime} \mathrm{W}\end{array}$ & $x$ & $x$ & $x$ & $x$ & $x$ & \\
\hline A. triste & I & USNMENT1430179 & Brazil & MGS & Agua Clara & MGS_8G & $\begin{array}{l}20^{\circ} 14^{\prime} \mathrm{S} \\
53^{\circ} 23^{\prime} \mathrm{W}\end{array}$ & $\times$ & $x$ & $x$ & & $x$ & \\
\hline A. triste & I & USNMENT1430180 & Brazil & MGS & Agua Clara & MGS_8H & $\begin{array}{l}20^{\circ} 14^{\prime} \mathrm{S}, \\
53^{\circ} 23^{\prime} \mathrm{W}\end{array}$ & $\times$ & $\times$ & $x$ & & $x$ & \\
\hline A. triste & I & USNMENT1430181 & Brazil & MGS & Agua Clara & MGS_8I & $\begin{array}{l}20^{\circ} 14^{\prime} \mathrm{S} \\
53^{\circ} 23^{\prime} \mathrm{W}\end{array}$ & $x$ & $\times$ & $x$ & $\times$ & & \\
\hline A. triste & I & USNMENT1430182 & Brazil & MGS & Agua Clara & MGS_8J & $\begin{array}{l}20^{\circ} 14^{\prime} \mathrm{S}^{\prime} \\
53^{\circ} 23^{\prime} \mathrm{W}\end{array}$ & $\times$ & $\times$ & $\times$ & $x$ & & \\
\hline A. triste & I & USNMENT1430183 & Brazil & MGS & Agua Clara & MGS_8K & $\begin{array}{l}20^{\circ} 14^{\prime} \mathrm{S} \\
53^{\circ} 23^{\prime} \mathrm{W}\end{array}$ & $x$ & $\times$ & $x$ & $x$ & & \\
\hline A. triste & 1 & USNMENT1430184 & Brazil & MGS & Agua Clara & MGS_8L & $\begin{array}{l}20^{\circ} 14^{\prime} \mathrm{S} \\
53^{\circ} 23^{\prime} \mathrm{W}\end{array}$ & $\times$ & $\times$ & $\times$ & & & \\
\hline A. triste & I & USNMENT1430185 & Brazil & MGS & Agua Clara & MGS_8M & $\begin{array}{l}20^{\circ} 14^{\prime} \mathrm{S} \\
53^{\circ} 23^{\prime} \mathrm{W}\end{array}$ & $x$ & $x$ & $x$ & & & \\
\hline A. triste & । & USNMENT1430186 & Brazil & MGS & Agua Clara & MGS_8N & $\begin{array}{l}20^{\circ} 14^{\prime} \mathrm{S} \\
53^{\circ} 23^{\prime} \mathrm{W}\end{array}$ & $\times$ & $x$ & $\times$ & $x$ & & \\
\hline A. triste & I & USNMENT1430187 & Brazil & MGS & Agua Clara & MGS_8O & $\begin{array}{l}20^{\circ} 14^{\prime} \mathrm{S} \\
53^{\circ} 23^{\prime} \mathrm{W}\end{array}$ & $x$ & $x$ & $x$ & & & \\
\hline A. triste & I & USNMENT1430188 & Brazil & MGS & Agua Clara & MGS_8P & $\begin{array}{l}20^{\circ} 14^{\prime} S^{\prime} \\
53^{\circ} 23^{\prime} \mathrm{W}\end{array}$ & $\times$ & $x$ & $x$ & $x$ & & \\
\hline A. triste & I & USNMENT1430189 & Brazil & MGS & Agua Clara & MGS_8Q & $\begin{array}{l}20^{\circ} 14^{\prime} \mathrm{S} \\
53^{\circ} 23^{\prime} \mathrm{W}\end{array}$ & $\times$ & $x$ & $x$ & $x$ & & \\
\hline A. triste & 1 & USNMENT01430159 & Brazil & Sao Paulo & Promissão & SP_AT2A & $\begin{array}{l}21^{\circ} 32^{\prime} \mathrm{S} \\
49^{\circ} 52^{\prime} \mathrm{W}\end{array}$ & $\times$ & & & & $x$ & \\
\hline A. triste & । & USNMENT01430160 & Brazil & Sao Paulo & Promissão & SP_AT2B & $\begin{array}{l}21^{\circ} 32^{\prime} \mathrm{S} \\
49^{\circ} 52^{\prime} \mathrm{W}\end{array}$ & $\times$ & & $x$ & & $x$ & \\
\hline A. triste & I & USNMENT01430161 & Brazil & Sao Paulo & Promissão & SP_AT2C & $\begin{array}{l}21^{\circ} 32^{\prime} \mathrm{S} \\
49^{\circ} 52^{\prime} \mathrm{W}\end{array}$ & $\times$ & & $x$ & & & \\
\hline A. triste & 1 & USNMENT01430162 & Brazil & Sao Paulo & Promissão & SP_AT2D & $\begin{array}{l}21^{\circ} 32^{\prime} \mathrm{S}, \\
49^{\circ} 52^{\prime} \mathrm{W}\end{array}$ & $x$ & & & & & \\
\hline A. triste & I & USNMENT01430163 & Brazil & Sao Paulo & Promissão & SP_AT2E & $\begin{array}{l}21^{\circ} 32^{\prime} \mathrm{S}, \\
49^{\circ} 52^{\prime} \mathrm{W}\end{array}$ & & & & & $x$ & \\
\hline A. triste & । & USNMENT01430164 & Brazil & Sao Paulo & Promissão & SP_AT2F & $\begin{array}{l}21^{\circ} 32^{\prime} \mathrm{S} \\
49^{\circ} 52^{\prime} \mathrm{W}\end{array}$ & $\times$ & & $x$ & & & $\times$ \\
\hline A. triste & I & USNMENT01430165 & Brazil & Sao Paulo & Promissão & SP_AT2G & $\begin{array}{l}21^{\circ} 32^{\prime} \mathrm{S} \\
49^{\circ} 52^{\prime} \mathrm{W}\end{array}$ & $x$ & & & & $x$ & \\
\hline A. triste & I & USNMENT01430166 & Brazil & Sao Paulo & Promissão & $\mathrm{SP} \_2 \mathrm{~A}$ & $\begin{array}{l}21^{\circ} 32^{\prime} \mathrm{S} \\
49^{\circ} 52^{\prime} \mathrm{W}\end{array}$ & & $x$ & & & & \\
\hline A. triste & I & USNMENT01430167 & Brazil & Sao Paulo & Promissão & $S P \_2 B$ & $\begin{array}{l}21^{\circ} 32^{\prime} \mathrm{S}, \\
49^{\circ} 52^{\prime} \mathrm{W}\end{array}$ & $\times$ & $x$ & & & $x$ & \\
\hline A. triste & । & USNMENT01430168 & Brazil & Sao Paulo & Promissão & SP_2C & $\begin{array}{l}21^{\circ} 32^{\prime} \mathrm{S} \\
49^{\circ} 52^{\prime} \mathrm{W}\end{array}$ & $\times$ & $x$ & $x$ & & & $\times$ \\
\hline A. triste & I & USNMENT01430169 & Brazil & Sao Paulo & Promissão & SP_13A & $\begin{array}{l}21^{\circ} 32^{\prime} \mathrm{S} \\
49^{\circ} 52^{\prime} \mathrm{W}\end{array}$ & $\times$ & $x$ & $x$ & & $x$ & \\
\hline A. triste & I & USNMENT01430170 & Brazil & Sao Paulo & Promissão & SP_13B & $\begin{array}{l}21^{\circ} 32^{\prime} \mathrm{S} \\
49^{\circ} 52^{\prime} \mathrm{W}\end{array}$ & & $x$ & $x$ & $x$ & & \\
\hline A. triste & I & USNMENT01430171 & Brazil & Sao Paulo & Promissão & SP_13C & $\begin{array}{l}21^{\circ} 32^{\prime} \mathrm{S} \\
49^{\circ} 52^{\prime} \mathrm{W}\end{array}$ & & $x$ & $x$ & & $x$ & \\
\hline
\end{tabular}


Table 1 Tick samples used for the molecular portion of this study and the gene amplifications results. Crosses indicate successful amplification and sequencing of the corresponding molecular marker (Continued)

\begin{tabular}{|c|c|c|c|c|c|c|c|c|c|c|c|c|c|}
\hline Species & $\mathrm{Mt}$ & Voucher ID & Country & $\begin{array}{l}\text { State/ province/ } \\
\text { department }\end{array}$ & Locality & Code & Coordinates & 125 & 165 & $\mathrm{DL}$ & ITS2 & $\cos 1$ & $\cos 2$ \\
\hline A. triste & I & USNMENT01430154 & Brazil & MGS & Brasilândia & MG_7A & $\begin{array}{l}21^{\circ} 16^{\prime} \mathrm{S} \\
51^{\circ} 51^{\prime} \mathrm{W}\end{array}$ & $x$ & $x$ & $x$ & $x$ & $\times$ & \\
\hline A. triste & I & USNMENT01430155 & Brazil & MGS & Brasilândia & MG_7B & $\begin{array}{l}21^{\circ} 16^{\prime} \mathrm{S} \\
51^{\circ} 51^{\prime} \mathrm{W}\end{array}$ & $\times$ & $x$ & $x$ & $x$ & $\times$ & \\
\hline A. triste & I & USNMENT01430156 & Brazil & MGS & Brasilândia & MG_7C & $\begin{array}{l}21^{\circ} 16^{\prime} \mathrm{S} \\
51^{\circ} 51^{\prime} \mathrm{W}\end{array}$ & & $\times$ & $x$ & $x$ & $\times$ & \\
\hline A. maculatum & $\|$ & & Colombia & Santander & Poima & SR_4A & $\begin{array}{l}6^{\circ} 09^{\prime} \mathrm{N}, \\
73^{\circ} 08^{\prime} \mathrm{W}\end{array}$ & & $x$ & $x$ & & $\times$ & \\
\hline A. triste & III & USNMENT01430102 & Mexico & Sonora & $\begin{array}{l}\text { Mina Mulatos, } \\
\text { Sahuaripa }\end{array}$ & $\mathrm{M} \times \_\mathrm{HO} 1$ & $\begin{array}{l}28^{\circ} 38^{\prime} \mathrm{N} \\
108^{\circ} 44^{\prime} \mathrm{W}\end{array}$ & $\times$ & $x$ & $x$ & $x$ & & \\
\hline A. triste & III & USNMENT01430102 & Mexico & Sonora & $\begin{array}{l}\text { Mina Mulatos, } \\
\text { Sahuaripa }\end{array}$ & $\mathrm{M} \times \_\mathrm{HO} 2$ & $\begin{array}{l}28^{\circ} 38^{\prime} \mathrm{N} \\
108^{\circ} 44^{\prime} \mathrm{W}\end{array}$ & $x$ & $x$ & $\times$ & $x$ & & \\
\hline A. maculatum & IV & USNMENT01430100 & Perú & Ica & Ica & PU_IC10A & $\begin{array}{l}14^{\circ} \mathrm{S} \\
75^{\circ} 46 \mathrm{~W}\end{array}$ & & $x$ & & & & \\
\hline A. maculatum & IV & USNMENT01430101 & Peru & Piura & Paimas & PU_PI1 & $\begin{array}{l}4^{\circ} 37^{\prime} \mathrm{S} \\
79^{\circ} 57^{\prime} \mathrm{W}\end{array}$ & $\times$ & $x$ & $x$ & & $\times$ & $\times$ \\
\hline A. maculatum & IV & USNMENT01430101 & Peru & Piura & Paimas & PU_PI2 & $\begin{array}{l}4^{\circ} 37^{\prime} \mathrm{S}, \\
79^{\circ} 57^{\prime} \mathrm{W}\end{array}$ & $\times$ & $x$ & $x$ & & $\times$ & $\times$ \\
\hline A. triste & IV & & Peru & Tumbes & $\begin{array}{l}\text { Pampas de } \\
\text { Hospital }\end{array}$ & PU_TU1 & $\begin{array}{l}3^{\circ} 41^{\prime} \mathrm{S}, \\
80^{\circ} 26^{\prime} \mathrm{W}\end{array}$ & $\times$ & $x$ & $\times$ & & $\times$ & \\
\hline A. triste & IV & & Peru & Tumbes & $\begin{array}{l}\text { Pampas de } \\
\text { Hospital }\end{array}$ & PU_TU2 & $\begin{array}{l}3^{\circ} 41^{\prime} \mathrm{S}, \\
80^{\circ} 26^{\prime} \mathrm{W}\end{array}$ & $\times$ & $x$ & $x$ & & & \\
\hline A. triste & IV & & Peru & Tumbes & $\begin{array}{l}\text { Pampas de } \\
\text { Hospital }\end{array}$ & PU_TU3 & $\begin{array}{l}3^{\circ} 41^{\prime} \mathrm{S}, \\
80^{\circ} 26^{\prime} \mathrm{W}\end{array}$ & $\times$ & $\times$ & $\times$ & $x$ & $\times$ & \\
\hline A. triste & I & & Uruguay & Canelones & EMA & CN_UY1 & $\begin{array}{l}34^{\circ} 44^{\prime} \mathrm{S}, \\
55^{\circ} 58^{\prime} \mathrm{W}\end{array}$ & $\times$ & $x$ & $x$ & $x$ & $x$ & \\
\hline A. triste & 1 & & Uruguay & Canelones & EMA & CN_UY2 & $\begin{array}{l}34^{\circ} 44^{\prime} \mathrm{S} \\
55^{\circ} 58^{\prime} \mathrm{W}\end{array}$ & $\times$ & $x$ & & $x$ & $\times$ & \\
\hline A. triste & III & USNMENT00865800 & USA & Arizona & $\begin{array}{l}\text { Atascosa Spring, } \\
\text { Santa Cruz Co. }\end{array}$ & AZ16_108 & $\begin{array}{l}31^{\circ} 24^{\prime} \mathrm{N} \\
111^{\circ} 10^{\prime} \mathrm{W}\end{array}$ & $\times$ & $x$ & $x$ & $x$ & & $\times$ \\
\hline A. triste & III & USNMENT00864570 & USA & Arizona & $\begin{array}{l}\text { Cave Canyon, } \\
\text { Santa Cruz Co. }\end{array}$ & AZ16_14 & $\begin{array}{l}31^{\circ} 43^{\prime} \mathrm{N} \\
110^{\circ} 47^{\prime} \mathrm{W}\end{array}$ & $\times$ & $\times$ & $x$ & $x$ & $\times$ & $x$ \\
\hline A. triste & III & USNMENT00865805 & USA & Arizona & $\begin{array}{l}\text { San Pedro } \\
\text { Riparian NCA, } \\
\text { Cochise Co. }\end{array}$ & AZ16_23 & $\begin{array}{l}31^{\circ} 33^{\prime} \mathrm{N} \\
110^{\circ} 08^{\prime} \mathrm{W}\end{array}$ & $\times$ & $\times$ & $x$ & $x$ & & \\
\hline A. triste & III & USNMENT00865805 & USA & Arizona & $\begin{array}{l}\text { San Pedro } \\
\text { Riparian NCA, } \\
\text { Cochise Co. }\end{array}$ & AZ16_40 & $\begin{array}{l}31^{\circ} 33^{\prime} \mathrm{N} \\
110^{\circ} 08^{\prime} \mathrm{W}\end{array}$ & $\times$ & $\times$ & $x$ & $x$ & $\times$ & $x$ \\
\hline A. triste & III & USNMENT00865805 & USA & Arizona & $\begin{array}{l}\text { San Pedro } \\
\text { Riparian NCA, } \\
\text { Cochise Co. }\end{array}$ & AZ16_121 & $\begin{array}{l}31^{\circ} 22^{\prime} \mathrm{N} \\
110^{\circ} 06^{\prime} \mathrm{W}\end{array}$ & $\times$ & $x$ & $x$ & $x$ & $\times$ & \\
\hline A. triste & III & USNMENT00865801 & USA & Arizona & $\begin{array}{l}\text { Gardner Canyon, } \\
\text { Santa Cruz Co. }\end{array}$ & AZ16_63 & $\begin{array}{l}31^{\circ} 42^{\prime} \mathrm{N} \\
110^{\circ} 47^{\prime} \mathrm{W}\end{array}$ & $\times$ & $\times$ & $x$ & $x$ & $\times$ & $\times$ \\
\hline A. triste & III & USNMENT00865800 & USA & Arizona & $\begin{array}{l}\text { Sycamore } \\
\text { Canyon, Santa } \\
\text { Cruz Co. }\end{array}$ & AZ16_1 & $\begin{array}{l}31^{\circ} 25^{\prime} \mathrm{N} \\
111^{\circ} 11^{\prime} \mathrm{W}\end{array}$ & $\times$ & $\times$ & & $x$ & & \\
\hline A. triste & III & USNMENT00865800 & USA & Arizona & $\begin{array}{l}\text { Sycamore } \\
\text { Canyon, Santa } \\
\text { Cruz Co. }\end{array}$ & AZ16_4 & $\begin{array}{l}31^{\circ} 25^{\prime} \mathrm{N} \\
111^{\circ} 11^{\prime} \mathrm{W}\end{array}$ & $\times$ & $x$ & $x$ & $x$ & & $\times$ \\
\hline A. maculatum & $\|$ & USNMENT1430199 & USA & Florida & Broward Co. & FL_24A & $\begin{array}{l}26^{\circ} 10^{\prime} \mathrm{N} \\
80^{\circ} 51^{\prime} \mathrm{W}\end{array}$ & $\times$ & $\times$ & $\times$ & $x$ & $x$ & $\times$ \\
\hline A. maculatum & $\|$ & USNMENT1430194 & USA & Georgia & Bulloch Co. & GA_19A & $\begin{array}{l}32^{\circ} 26^{\prime} \mathrm{N} \\
81^{\circ} 51^{\prime} \mathrm{W}\end{array}$ & $\times$ & $x$ & $x$ & $x$ & $x$ & \\
\hline
\end{tabular}


Table 1 Tick samples used for the molecular portion of this study and the gene amplifications results. Crosses indicate successful amplification and sequencing of the corresponding molecular marker (Continued)

\begin{tabular}{|c|c|c|c|c|c|c|c|c|c|c|c|c|c|}
\hline Species & Mt & Voucher ID & Country & $\begin{array}{l}\text { State/ province/ } \\
\text { department }\end{array}$ & Locality & Code & Coordinates & 125 & 165 & $\mathrm{DL}$ & ITS2 & $\operatorname{cox} 1$ & $\cos 2$ \\
\hline A. maculatum & $\|$ & USNMENT1430195 & USA & Georgia & Bulloch Co. & GA_19B & $\begin{array}{l}32^{\circ} 26^{\prime} \mathrm{N} \\
81^{\circ} 51^{\prime} \mathrm{W}\end{array}$ & $x$ & & & & $x$ & \\
\hline A. maculatum & $\|$ & USNMENT1430196 & USA & Georgia & Bulloch Co. & GA_20A & $\begin{array}{l}32^{\circ} 26^{\prime} \mathrm{N} \\
81^{\circ} 51^{\prime} \mathrm{W}\end{array}$ & $x$ & $x$ & $x$ & $x$ & $x$ & \\
\hline A. maculatum & $\|$ & USNMENT1430197 & USA & Georgia & Bulloch Co. & GA_21A & $\begin{array}{l}32^{\circ} 26^{\prime} \mathrm{N} \\
81^{\circ} 51^{\prime} \mathrm{W}\end{array}$ & $x$ & $x$ & $\times$ & $x$ & $x$ & \\
\hline A. maculatum & $\|$ & USNMENT1430198 & USA & Georgia & Bulloch Co. & GA_21B & $\begin{array}{l}32^{\circ} 26^{\prime} \mathrm{N}, \\
81^{\circ} 51^{\prime} \mathrm{W}\end{array}$ & $x$ & $x$ & $x$ & & $x$ & \\
\hline A. maculatum & $\|$ & USNMENT1430199 & USA & Georgia & Bulloch Co. & GA_21C & $\begin{array}{l}32^{\circ} 26^{\prime} \mathrm{N} \\
81^{\circ} 51^{\prime} \mathrm{W}\end{array}$ & $x$ & $x$ & $x$ & $x$ & $x$ & \\
\hline A. maculatum & $\|$ & USNMENT1430200 & USA & Georgia & Bulloch Co. & GA_21D & $\begin{array}{l}32^{\circ} 26^{\prime} \mathrm{N} \\
81^{\circ} 51^{\prime} \mathrm{W}\end{array}$ & $x$ & $x$ & $\times$ & & $x$ & \\
\hline A. maculatum & $\|$ & USNMENT1430189 & USA & Georgia & Camden Co. & GA_23A & $\begin{array}{l}30^{\circ} 58^{\prime} \mathrm{N} \\
81^{\circ} 42^{\prime} \mathrm{W}\end{array}$ & $x$ & $x$ & $x$ & $x$ & $x$ & \\
\hline A. maculatum & $\|$ & USNMENT1430184 & USA & Georgia & Monroe Co. & GA_22A & $\begin{array}{l}33^{\circ} 02^{\prime} \mathrm{N} \\
83^{\circ} 44^{\prime} \mathrm{W}\end{array}$ & $\times$ & $x$ & $x$ & $x$ & $x$ & $\times$ \\
\hline
\end{tabular}

Abbreviations: BA Buenos Aires, SDE Santiago del Estero, na not applicable

external spur long and sharp, internal spur as a small tubercle; coxae II-IV each with a triangular, short blunt spur; trochanters without spurs. Genital aperture U-shaped (Figs. 2h, 4g, 5f, 6f).

\section{Morphotype I: Amblyomma triste Koch, 1844 (sensu stricto)}

Distribution: Present study: Argentina, Paraguay, southern Brazil and Uruguay.

Type- and voucher material examined: Type-specimens of A. triste (ZMB 1046): 2 우, Montevideo, Uruguay. Voucher specimens: Argentina: Buenos Aires Province: 5우우 5새, Reserva Otamendi (INTA 1978); 30 우우 $30 \widehat{\jmath}$, Inta Delta del Paraná (INTA 1979, 2009, 2033, 2042, 2065, 2104, 2115, 2198); 1 \% $\hat{\delta}$, Punta Indio

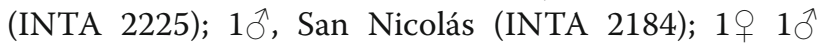
Dársena Paraná Guazú (INTA 2094); Corrientes Province: 1 ㅇ (USNMENT00958436); 1 우 Estero del Iberá (INTA 1914); 1 우 1ठ․, Estancia Rincón del Socorro (INTA 2129); Entre Ríos Province: $1 \hat{\jmath}$, Villa Paranacito (INTA 2194); 1 ㅇ 1소, Isla Paloma (INTA 2114); Formosa Province: 1 우 1 $\hat{0}$, Reserva El Bagual (INTA 2096); 19 Colonia Pastoril (INTA 2151). Bolivia: Beni Department, 1오. Pampa de Mais (USNMENT00958430). Brazil: State of Mato Grosso do Sul: $1 \propto 1{ }^{\lambda}$, Bataguassu

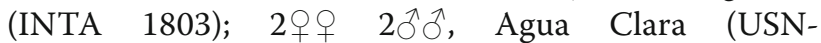
MENT01430164); State of São Paulo: 3 우 $3 \hat{\jmath} \hat{o}$, Promissão (USNMENT01430159); 1ㅇ, Porto Primavera (USNMENT00958438). Paraguay: locality not indicated, 1 ㅇ $1 \hat{\jmath}$, (USNMENT00958439); Boquerón: $3 \hat{\partial} \widehat{\partial}$ Estancia Faro Moro (USNMENT00958431). Uruguay:

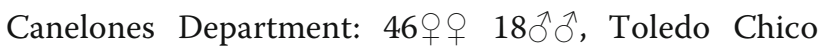

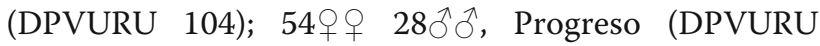
149); Montevideo Department: 45 웅 $33 \hat{\jmath} \widehat{\partial}$, Villa García, (DPVURU 181); San José Department: 5 우우 $2 \hat{\jmath} \widehat{\jmath}$, Playa Pascual (DPVURU 117).

\section{Description (Fig. 2a-h)}

Male. Chitinous scutes on ventral surface of festoons well developed (Fig. 2a), visible dorsally (Fig. 2b), sometimes lined with whitish enamel (Fig. 2c, f). Postero-median spot wider than enameled stripe between postero-median and postero-lateral spots (Fig. 2b). One spine and one fine seta present on tibiae of legs II-IV (Fig. 2e). Spiracular plates almost oval, with short, wide dorsal projection (Fig. 2f), as wide as adjoining festoon, spurs on coxa IV long and sometimes reaching anus (Fig. 2a).

Female. Brown central spot extending to the posterior margin of the scutum present (Fig. 2d). One spine and one seta on tibiae of legs II-IV present as in male. Spiracular plates usually oval, with short, wide dorsal projection longer than in males, dorsal projection as wide as adjoining festoon (Fig. 2g).

\section{Morphotype II: Amblyomma maculatum Koch, 1844 (sensu stricto)}

Distribution: Present study: Colombia, Guatemala, Honduras, Mexico, Nicaragua, USA and Venezuela.

Type- and voucher material examined: Type-specimen of A. maculatum (ZMB 1044): 10, "Carolina", USA 

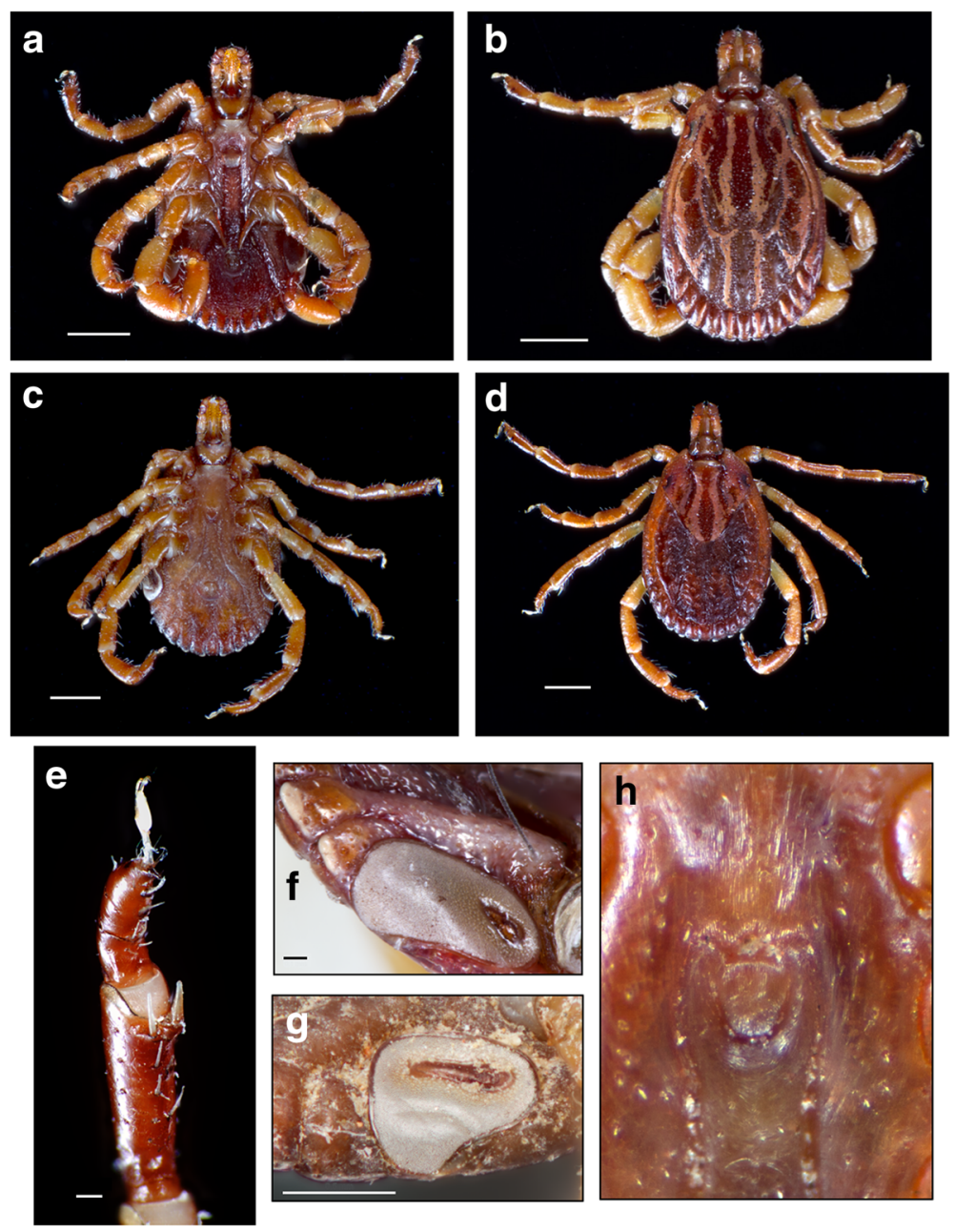

Fig. 2 Morphotype I: Amblyomma triste (sensu stricto). Male, ventral (a) and dorsal (b) views; female, ventral (c) and dorsal (d) views; tibiae of legs II-IV (e); spiracular plates in the male (f) and female $(\mathbf{g})$, and genital aperture (h). Scale-bars: $\mathbf{a}, 1 \mathrm{~mm} ; \mathbf{b}, 1 \mathrm{~mm} ; \mathbf{c}, 1 \mathrm{~mm} ; \mathbf{d}, 1 \mathrm{~mm} ; \mathbf{e}, 0.1 \mathrm{~mm} ; \mathbf{f}, 0.1$ $\mathrm{mm} ; \mathbf{g}, 0.5 \mathrm{~mm}$

(presumably from one of the Carolinas). Colombia: Chachagüí Department: 3 우 $1 \hat{\jmath}$, Pasto (USNMENT00957339);

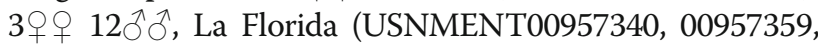
00957329); Valle del Cauca Department: 6 우 $3 \hat{\jmath} \hat{o}$ Cali (USNMENT00957349, 00957344, 00957342, 00957325. Guatemala: Chimaltenango Department: $1 \delta^{\lambda}$, Acatenango (USNMENT00957404). Honduras: Atlántida Department:

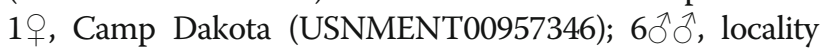
not specified (USNMENT957405). Mexico: Sinaloa Department: 1 우, Culiacan (USNMENT00957402); 5 우우 $7 \hat{\jmath} \widehat{\jmath}$ Los Mochis (USNMENT00957387, 00957378). Nicaragua: Carazo Department: $1 \hat{\delta}$, Dolores (USNMENT00957406); Rivas Department: 1 ${ }^{\lambda}$, Rivas (USNMENT957408). USA:

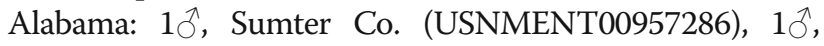
Houston Co. Dothan Landmark Park (USNMENT 00957256); Florida: 1 우 $2 \hat{\jmath} \hat{\jmath}$, Orange Co, Orlando (USNMENT00957220), 3 우 $1 \delta^{\lambda}$, Palm Beach (USNMENT 00957250), $2 \widehat{\partial}$, Marion Co, Ocala (USNMENT0095
7350), 1 우 $7 \lesssim \precsim$, Collier Co, Bear Island (USN-

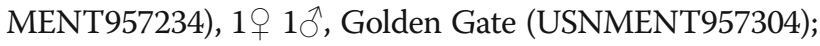
Georgia: $3 q++1 \delta^{\lambda}$, Grady Co, Cairo, (USNMENT0095 7242); 29 우 $4 \overbrace{}^{\hat{\gamma}}$, Thomas Co, Thomasville (USNMENT 00957384), 10ิ, Bulloch Co, Statesboro (USNMENT 00957364), 3우우, Decatur Co, Bainbridge (USNMEN T00957290), 1운, Lowndes Co. Moodyfields Airforce Base (USNMENT00957310), 19, Liberty Co, St. Catherine Isl. (USNMENT00957334); Indiana: 10ิ, Hamilton Co, Carmel, (USNMENT00957317); Louisiana: 4 우 $91{ }^{\lambda}$, Cameron Co, Johnson's Bayou (USNMENT00957271), 1우 20ㅊㅇ, Cameron (USNMENT00957280), $192 \hat{\jmath}$, Baton Rouge (USNMENT00957373, 00957353); Mississippi: $2 \widehat{\partial}$ Pearl River Co, Mc Neill (USNMENT00957372), 3우, Poplarville (USNMENT00957273), 10, Prentiss Co, Booneville

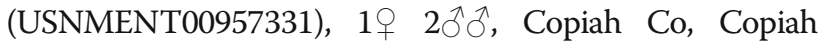
(USNMENT00863742, 00957332), 10َ, Covington Co, Collins (USNMENT00957281), 1q Hinds Co, Jackson 

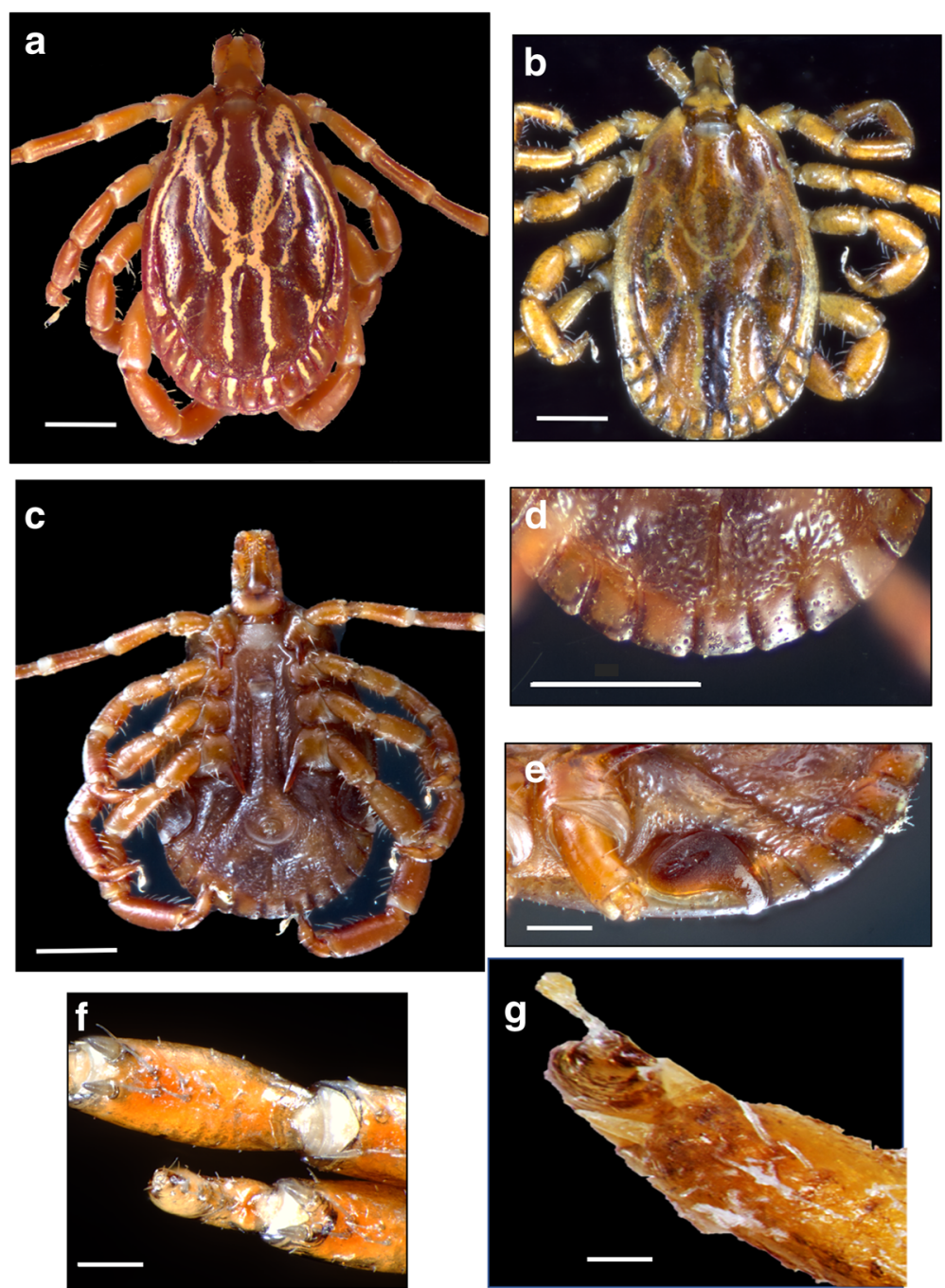

Fig. 3 Morphotype II: Amblyomma maculatum (sensu stricto). Male, dorsal (a, USA; b, Venezuela) and ventral (c, d, USA) views; male, spiracular plates (e, USA); tibiae of legs II-IV in the male (f, USA) and female (g, USA). Scale-bars: a, $1 \mathrm{~mm} ; \mathbf{b}, 1 \mathrm{~mm} ; \mathbf{c}, 1 \mathrm{~mm} ; \mathbf{d}, 1 \mathrm{~mm} ; \mathbf{e}, 0.5 \mathrm{~mm} ; \mathbf{f}, 0.1 \mathrm{~mm}$; g, $0.1 \mathrm{~mm}$

(USNMENT00957334), 2 우, Forest Co. (USNMENT0095 7302); Oklahoma: 19, Pittsburg Co, Weathers (USNMENT00957366), 2qo , Mayes Co, Chouteau (USNMENT00957356), $2 \overbrace{}^{\lambda} 2$ 우, Osage Co. (USNMENT00 957292). State of South Carolina: $3 \hat{\partial} \delta$ 1우, Charleston Co, Charleston (USNMENT00957380), 6우우, Johns Isl. (USNMENT00957283), $1 \delta^{\lambda} 1$, , Allendale Co, Allendale (USNMENT00957266); 2qㅇ, Georgetown Co, Georgetown (USNMENT00957270), 1今, Jasper Co, Ridgeland (USNMENT00957246). Texas: 20, Dallas (USNMENT00 957239, 00957385), 17우, Galveston Co, High Island

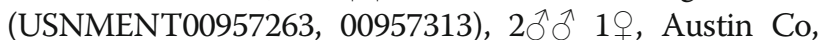
Sealy (USNMENT00957232), 35 $\hat{\jmath} \widehat{\jmath} 9$ 우우, Cat Springs (USNMENT00863706, 00957375, 00957370), $2 \widehat{ } \sigma^{\lambda} \mathrm{New}$

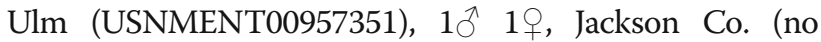

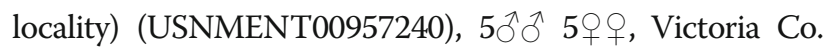
Victoria (USNMENT00957237, 00957377), 2 우, Nueces Co, Corpus Christi (USNMENT00957382), 50 어 1우, Fayette Co, Shulenberg (USNMENT00957381), 16ㅊㅎㅅ 6우우, Jim Hogg Co, Hebbronville (USNMENT00957288). Venezuela: State of Cojedes: $2 \hat{\jmath} \widehat{\partial}$, Cojedes (USNMENT 00957347); State of Miranda: 1오․ Alto de Pipe (USNMENT00957362); State of Monagas: 2 우 $1^{\hat{\jmath}}$, San Agustin (USNMENT00957337, 00957333).

\section{Description (Figs. 3a-g, 4a-i)}

Male. Chitinous scutes present on ventral surface of festoons, often less salient than in Morphotype I, sometimes reduced to a posteromedian tubercle (Fig. 3c-e). 

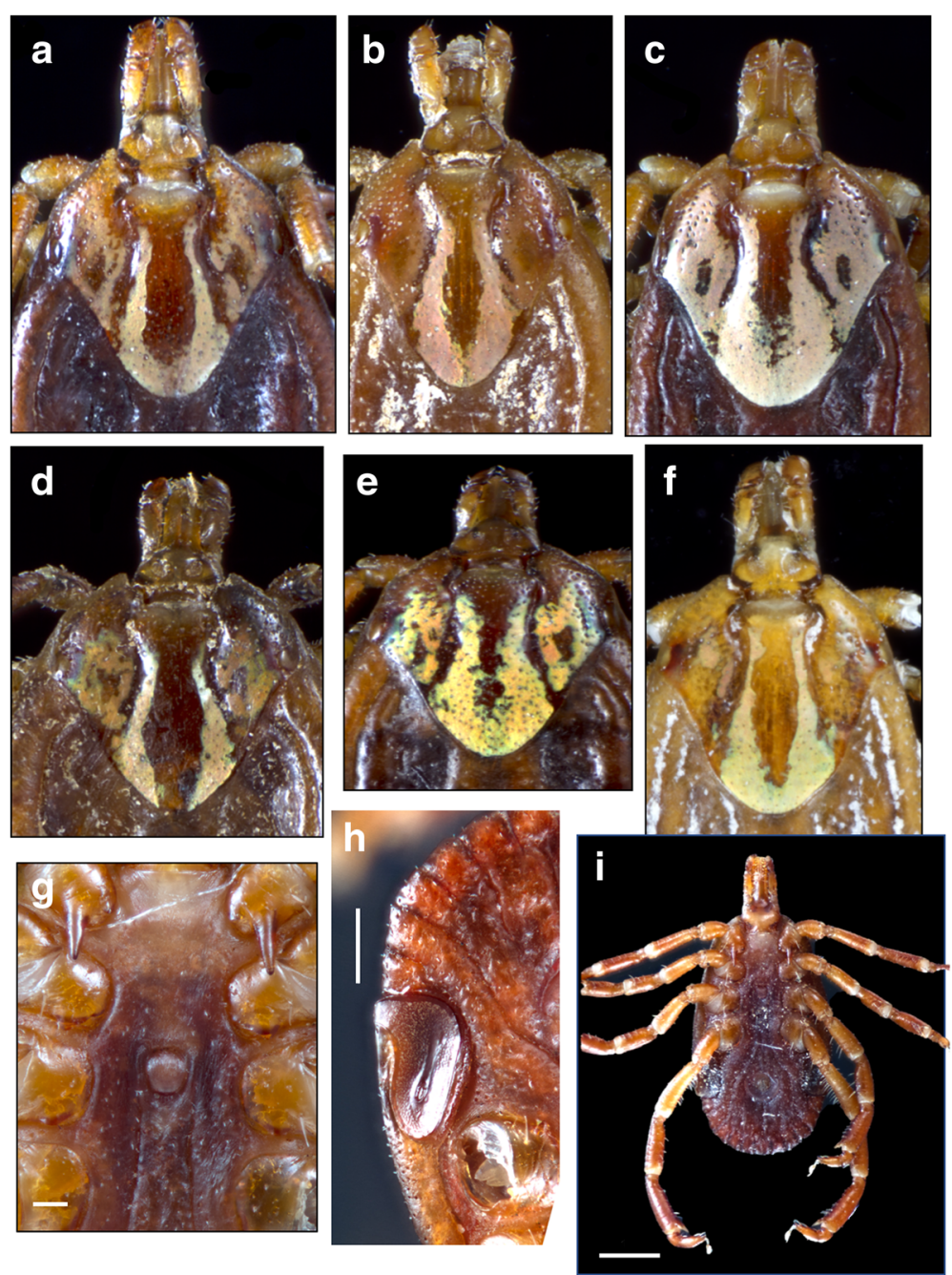

Fig. 4 Morphotype II: Amblyomma maculatum (sensu stricto). Female, variation in dorsal ornamentation (a-e, USA; $\mathbf{f}$, Venezuela); genital aperture (g, USA). Male, spiracular plates (h, USA); male, ventral view (i, USA). Scale-bars: $\mathbf{g}, 0.2 \mathrm{~mm} ; \mathbf{h}, 0.5 \mathrm{~mm} ; \mathbf{i}, 1 \mathrm{~mm}$

Two robust spines almost equal in size present on tibiae of legs II-IV (Fig. 3f). Spiracular plates comma-shaped, with tip of dorsal projection equal in width or slightly narrower than half of adjacent festoon; dorsal projection perpendicular to longitudinal axis of spiracular plate (Fig. 3e). Spurs on coxa IV not reaching anus (Fig. 3c); postero-median spot wider than enameled stripe between postero-median and postero-lateral spots (Fig. 3a, b).

Female. Two spines almost equal in size present on tibiae of legs II-IV (Fig. 3g). Spiracular plates comma-shaped, with dorsal projection perpendicular to axis of spiracular plate, as wide or slightly wider than half-festoon width (Fig. 4h). Scutal brown central area long and narrow, not reaching posterior margin of scutum in most specimens, a few specimens with central area reaching posterior margin of the scutum; enameled surface of scutum variable in shape and proportions (Fig. 4a-f).

\section{Morphotype III: Amblyomma sp. cf. A. triste - A. maculatum}

Distribution: Present study: northern Mexico and Arizona, USA.

Voucher material examined: Mexico: State of Sonora,

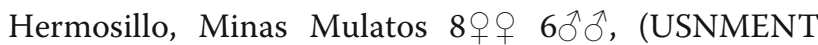
01430102), 1 $\hat{\jmath}$, Locality not specified (USNMENT 958432). USA: Arizona: $191{ }^{\widehat{\gamma}}$, Cochise Co, San Pedro Riparian National Conservation Area (USNMENT00865805), 1우 1今̂, Santa Cruz Co. Santa Rita Mountains, Gardner Canyon (USNMENT00865801), 1910 , Santa Cruz Co, Pajarita Wilderness Area (USNMENT00865800), $1910 \hat{\jmath}$, Santa Cruz Co, Santa Rita Mountains, Cave Canyon (USNMENT00864570), 1 우 1 $0^{\lambda}$, Cochise Co, San Pedro Riparian Conservation Area, Herford Road (USNMENT00864571) and all ticks used in [23]. 

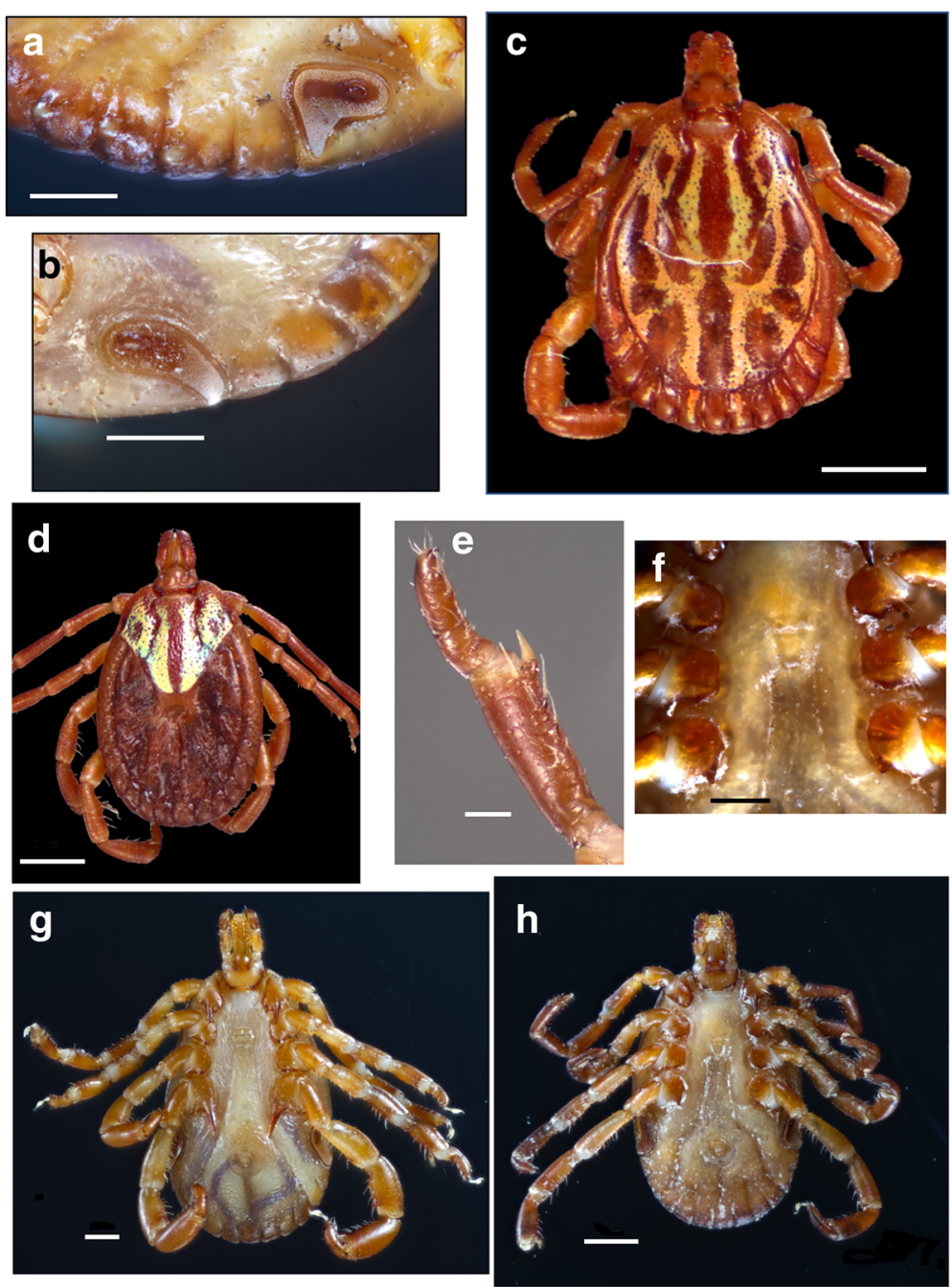

Fig. 5 Morphotype III: Amblyomma sp. cf. A. triste - A. maculatum [Arizona (USA) and Mexico]. Female, spiracular plate (a); male, spiracular plate (b); male, dorsal view (c); female, dorsal view (d); tibiae legs II-IV (e); female, genital aperture (f); male, ventral view (g); female, ventral view (h). Scale-bars: a, $0.5 \mathrm{~mm} ; \mathbf{b}, 0.5 \mathrm{~mm} ; \mathbf{c}, 1 \mathrm{~mm} ; \mathbf{d}, 1 \mathrm{~mm} ; \mathbf{e}, 0.1 \mathrm{~mm} ; \mathbf{f}, 0.5 \mathrm{~mm} ; \mathbf{g}, 0.5 \mathrm{~mm} ; \mathbf{h}, 1 \mathrm{~mm}$

\section{Description (Fig. 5a-h)}

Male. Chitinous scutes present on ventral surface of festoons (Fig. 5g), visible dorsally (Fig. 5a-c). Postero-median spot wider than enameled stripe between postero-median and postero-lateral spots in most specimens (Fig. 5c), a few specimens with postero-median spot almost equal in width to stripe between postero-median and postero-lateral spots. One spine and one seta present on tibiae of legs II-IV (Fig. 5e). Spiracular plates comma-shaped, with dorsal projection approximately half as wide as adjoining festoon; dorsal projection as an extension of spiracular plate, not perpendicular to its axis (Fig. 5b). Spurs on coxa IV not reaching anus (Fig. 5g).

Female. Brown scutal central area long and narrow, always reaching posterior margin of scutum (Fig. $5 \mathrm{~d}$ ). One spine and one seta present on tibiae of legs II-IV as in male. Spiracular plates with dorsal projection perpendicular to plate axis, as wide or narrower than a third of adjoining festoon (Fig. 5a).

\section{Morphotype IV: Amblyomma sp. cf. A. triste - A. maculatum}

Distribution: Present study: Chile, Ecuador and Peru. Voucher material examined: Chile: XV Arica and Pari-

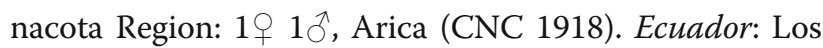
Ríos Province: $1 \hat{\jmath}$, locality not indicated (USNMENT00958433); Guayas Province: 10̂, "litoral region" (USNMENT00958435); 1 $\widehat{\partial}$, Naranja (USNMENT0095 8428); El Oro Province: 1옹 Machala (USNMENT00 957381). Peru: Ica Department: 1 , Ica (USNMENT01 430100); Piura Deparment: 1910 , Piura (USNMENT01 430101). Images taken after DNA was extracted from specimens. 

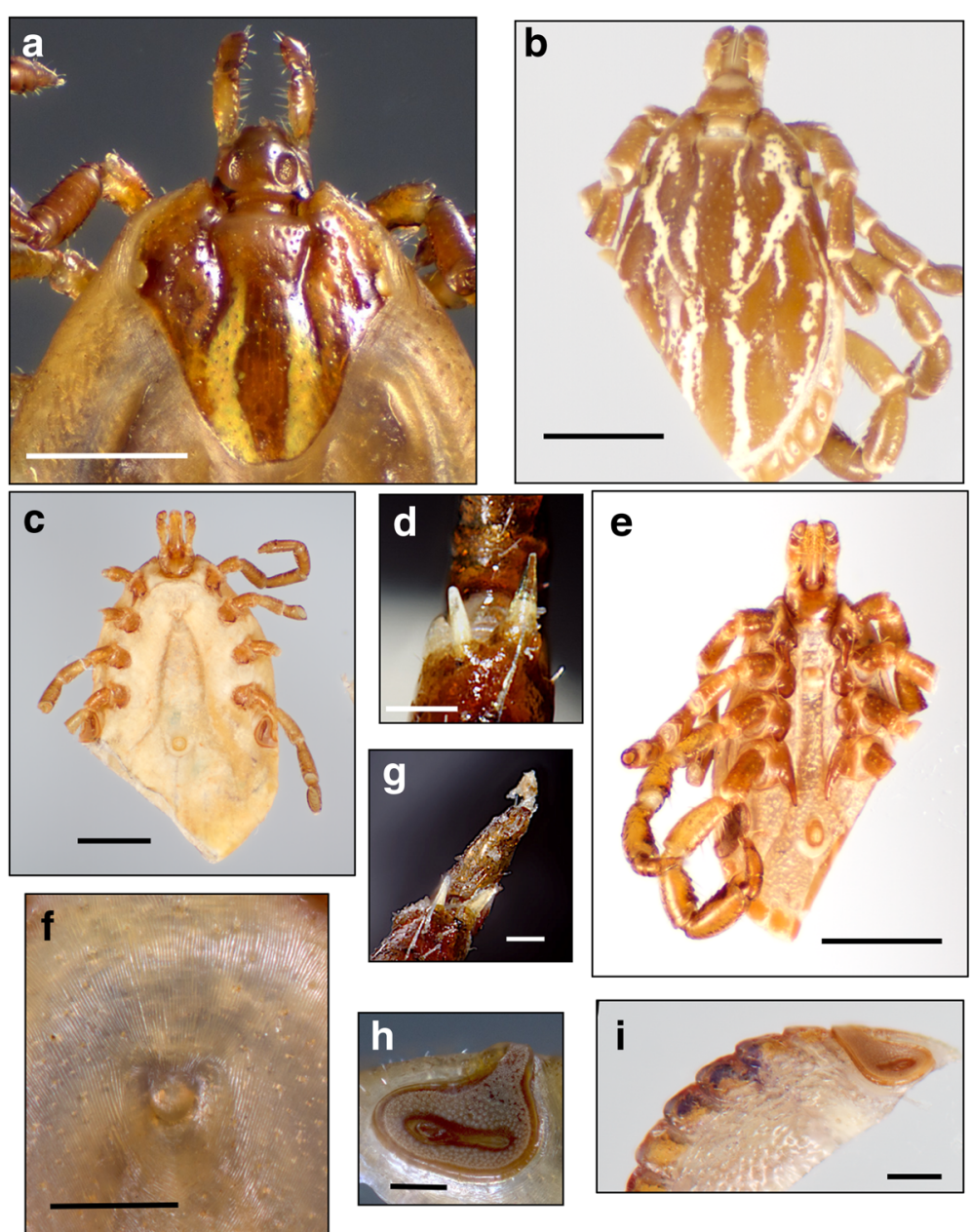

Fig. 6 Morphotype IV: (Peru) Amblyomma sp. cf. A. triste - A. maculatum (Peru). Female, scutum (a); male, dorsal view (b); tibiae of legs II-IV (d, g); male, ventral view (e); female, genital aperture (f); female, spiracular plates (h, i). $\mathbf{a}, 1 \mathrm{~mm} ; \mathbf{b}, 1 \mathrm{~mm} ; \mathbf{c}, 1 \mathrm{~mm} ; \mathbf{d}, 0.1 \mathrm{~mm} ; \mathbf{e}, 1 \mathrm{~mm} ; \mathbf{f}, 0.5 \mathrm{~mm} ; \mathbf{g}, 0.1$ $\mathrm{mm} ; \mathbf{h}, 0.5 \mathrm{~mm}, \mathbf{i}, 0.5 \mathrm{~mm}$

\section{Description (Fig. 6a-i)}

Male. Chitinous scutes present on ventral surface of festoons (Fig. 6e), visible dorsally (Fig. 6b). Two spines slightly different in size present on tibiae of legs II-IV but originating at a different level along tarsi and therefore appearing to be of different lengths (Fig. 6d). Spiracular plates comma-shaped, with dorsal projection about half as wide as adjoining festoon extending from plate at an obtuse angle (Fig. 6i). Postero-median spot wider than enameled stripe between postero-median and postero-lateral spots (Fig. 6b).

Female. Central scutal area long, narrow (Fig. 6a), reaching posterior margin of scutum in most specimens. Spines on tibiae of legs II-IV as in males (Fig. 6g). Spiracular plates comma-shaped, with dorsal projection perpendicular to plates and approximately half as wide as adjoining festoon.

\section{PCR amplification and sequence alignment}

Although we did not obtain all gene sequences for each sample, we obtained sequences representative for all species and morphotypes (Table 1). The lengths of the data matrices were as follows: $338 \mathrm{bp}$ for $12 S \mathrm{rDNA}, 410 \mathrm{bp}$ for $16 S$ rDNA, 497 bp for cox1, 368 bp for DL, and 954 bp for ITS2. The concatenated mitochondrial dataset (mtDNA) included $1604 \mathrm{bp}$, the mt+nDNA $2556 \mathrm{bp}$, and the alignment for molecular clock and dating analyses 1469 bp (after removing regions that were too variable between the $A$. maculatum and the $A$. cajennense groups for unambiguous alignment). GenBank accession numbers for sequences generated in the present study are: KU284849-KU284860, MG076929-MG076938 (A. maculatum $12 S$ rDNA); KU284861, KU284862 (A. neumanni $12 S$ rDNA); KU284863, KU284864 (A. parvitarsum $12 S$ rDNA); KU284865-KU284921 (A. triste $12 S$ rDNA); KU28 4922-KU284929 (A. tigrinum $12 S$ rDNA); KU284930KU284941, KU284999, MG076911-MG076919 (A. 
maculatum $16 S$ rDNA); KU284942-U284998, KU285000, KU285001 (A. triste 16S rDNA); KU285002-KU285010 (A. tigrinum $16 S$ rDNA); KU285011 (A. neumanni $16 S \mathrm{rDNA}$ ); KU285012 (A. parvitarsum $16 S$ rDNA); KU285013KU285024 (A. maculatum DL); KU285025-KU285079 ( $A$. triste DL); KU285080-KU285086 (A. tigrinum DL); KU285087 (A. neumanni DL); KU285088-KU285094, MG076920-MG076928 (A. maculatum ITS2); KU285095KU285137 (A. triste ITS2); KU285138, KU285139 (A. tigrinum ITS2); KU306550-KU306598 (A. triste cox1); KU306599 (A. neumanni cox1); KU306600 (A. parvitarsum cox1); KU302492-KU302504, MG251313 (A. maculatum cox1); KU302505-KU302511 (A. tigrinum cox1); KU306601-KU306604 (A. maculatum cox2); KU306605-KU306607 (A. tigrinum cox2); KU306608KU306611 (A. triste cox2); KU306612 (A. parvitarsum); KU306613 (A. neumanni cox2). Figure 1 illustrates the sites where the ticks used for phylogenetic analyses were collected.

\section{Sequences and haplotype diversity}

If we exclude the outgroup sequences, the $8712 S \mathrm{rDNA}$ gene sequences were represented by 17 unique haplotypes (Additional file 1: Table S1), the $9116 S$ rDNA sequences by 34 unique haplotypes (Additional file 2: Table S2), the $73 \operatorname{cox} 1$ sequences by 32 unique haplotypes (Additional file 3: Table S3), the 81 DL sequences by 40 unique haplotypes (Additional file 4: Table S4) and the 62 ITS2 sequences by 15 unique genotypes (Additional file 5: Table S5). A total of 42 haplotypes were unique among the 63 concatenated mtDNA sequences, and 27 among the 31 concatenated $\mathrm{mt}+\mathrm{nDNA}$ datasets. For all mitochondrial datasets, haplotypes were consistently shared by ticks corresponding to Morphotypes II and III. Otherwise, no haplotypes were shared between other morphotypes.

\section{Phylogenetic analyses \\ Separate datasets}

The separate gene sequence analyses (phylogenies not shown) were characterized by overall limited resolution. The ingroup was consistently strongly supported. Amblyomma tigrinum was always monophyletic, mostly as the sister lineage to the A. maculatum - A. triste cluster (16S rDNA, DL and ITS2), but also as the sister group of Peruvian Morphotype IV (cox1) or embedded within Morphotype I ( $12 S \mathrm{rDNA})$. The overall structure of the five phylogenies was different, with Morphotypes II and III never clustering in two separate lineages, while the southern South American and the Peruvian sequences were sometimes identifiable as distinct clades (cox1). In addition, the analysis of ITS2 sequences only identified a supported polytomic ingroup. A closer examination of the ITS2 sequences revealed that when the matrix included outgroups, informative characters were 108 (11\%); however, when outgroup sequences were excluded, informative characters only represented $0.9 \%$. In the ITS2 ingroup matrix substitutions were mostly randomly scattered singleton mutations and indels with no phylogenetic information.

\section{Mitochondrial concatenated datasets (12S rDNA + 16S $r D N A+\operatorname{cox} 1+D L)$}

The MP (Maximum Parsimony) analysis of the mtDNA matrix found 6586 equally parsimonious trees (length = 506). The resolution of the MP strict consensus tree was better than that recovered for the separate datasets. The A. tigrinum and the A. maculatum - A. triste clusters were sister clades and both were well supported. Within the A. maculatum - A. triste clade, the first split occurred between Morphotype IV (Peru) and the remaining lineages. Morphotypes II and III were intermixed within a single clade, except for one sequence (identified as CO-US/GA in the tree) which corresponded to a Colombian sequence. Morphotype I was sister to Morphotypes II+III and included all southern South American sequences. Bayesian analysis recovered an almost identical topology (Fig. 7). Within these supported clades, resolution was very limited and when monophyletic, clades did not correspond to any geographical or ecologically meaningful subset.

\section{Mitochondrial-nuclear concatenated dataset (12S rDNA + $16 S$ rDNA + cox $1+D L+I T S 2)$}

The concatenated dataset represented a matrix of $2556 \mathrm{bp}$ (31 sequences, corresponding to 27 unique haplotypes and one outgroup). The MP analysis identified a total of 56 equally parsimonious trees (length $=770$ ). The MP and Bayesian inference BI reconstructions were almost completely congruent: the ingroup was monophyletic and $A$. tigrinum was consistently identified as the supported sister clade to the monophyletic $A$. maculatum - A. triste clade (Fig. 7b). However, by MP, the A. maculatum - A. triste clade was polytomic leading to monophyletic Morphotypes I, II+III, and IV. While Morphotype II was found to be monophyletic, Morphotype III did not cluster in a single supported clade. By BI, Morphotype IV was found to be the sister clade to the remaining morphotypes which clustered in a well-supported lineage (Fig. 7b). Morphotype I was monophyletic and, again, the structure within Morphotype I did not appear to correspond to geographical subdivisions with sequences from different regions represented in each clade. The last monophyletic clade included a monophyletic Morphotype II and a paraphyletic Morphotype III (Fig. 7b).

Divergence values in the $\mathrm{mt}+\mathrm{nDNA}$ dataset, calculated after the best mutation model was evaluated by ModelTest, showed that the distance between outgroup and 


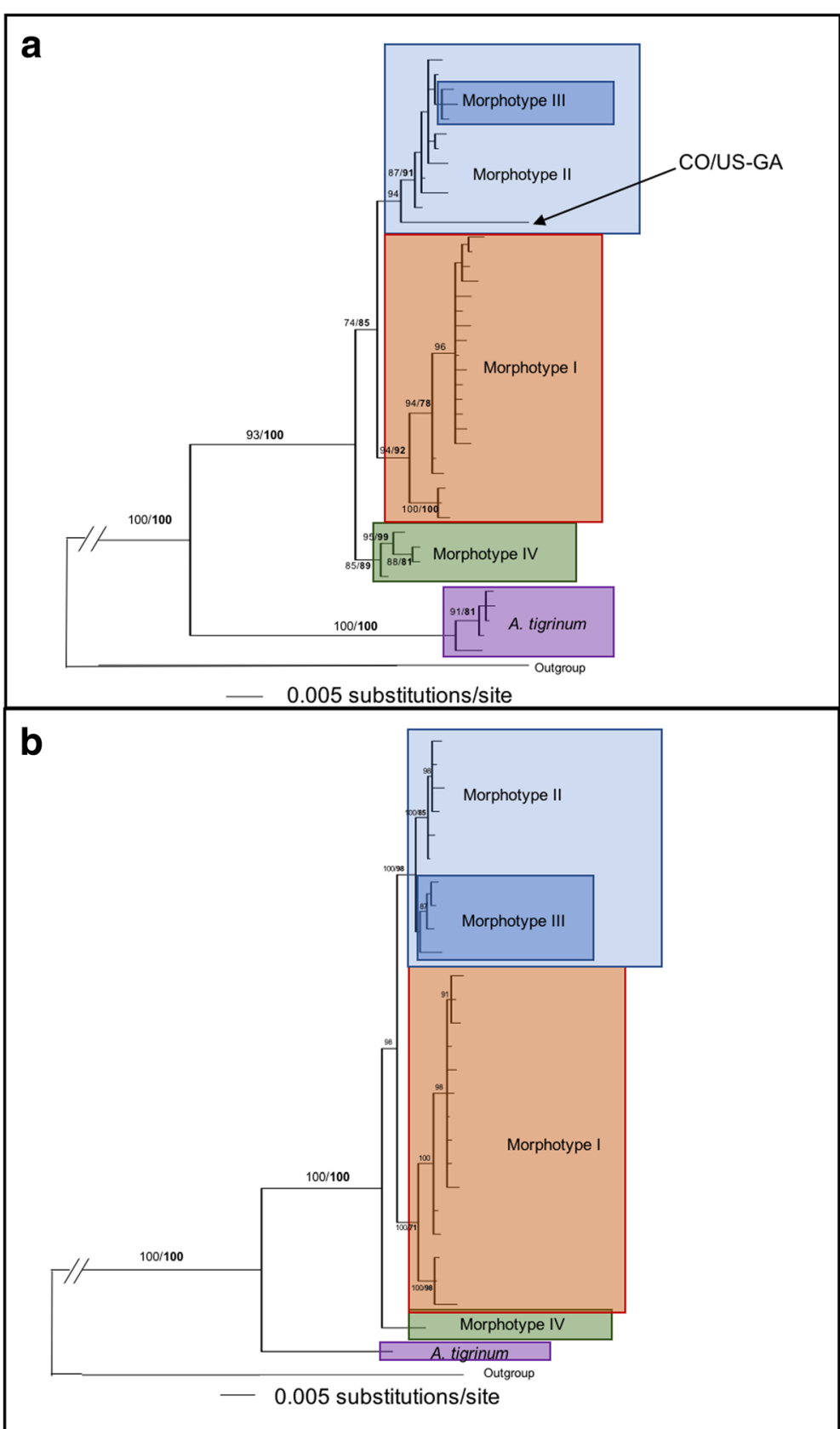

Fig. 7 Phylogenetic reconstruction of the mitochondrial concatenated dataset (a), and the total evidence (mitochondrial + nuclear) concatenated dataset (b). Trees were inferred by Bayesian analysis. Numbers on branches represent maximum parsimony bootstrap support and Bayesian posterior probability support (in bold)

ingroup varied from 18.60 to $19.01 \%$, between A. tigrinum and A. maculatum - A. triste by $3.91-4.31 \%$, between Morphotype IV and the remaining clades by $1.22-1.70 \%$, between Morphotype I and II+III by $0.91-1.22 \%$ and between Morphotype II and III by $0.32-0.71 \%$. Within Morphotype II, distances varied between 0 and $0.28 \%$, within Morphotype III between 0 and $0.51 \%$ and within Morphotype I between 1.22 and $1.70 \%$.

\section{Node dating}

DAMBE analyses revealed no significant mutation rate differences between the main ingroup lineages $(P=$ 0.053-0.9) including A. tigrinum. Both the least-square method and the likelihood ratio test did not reject the molecular clock hypothesis, further confirming that the lineages under consideration did not evolve at significantly different rates. Although this would justify the use 


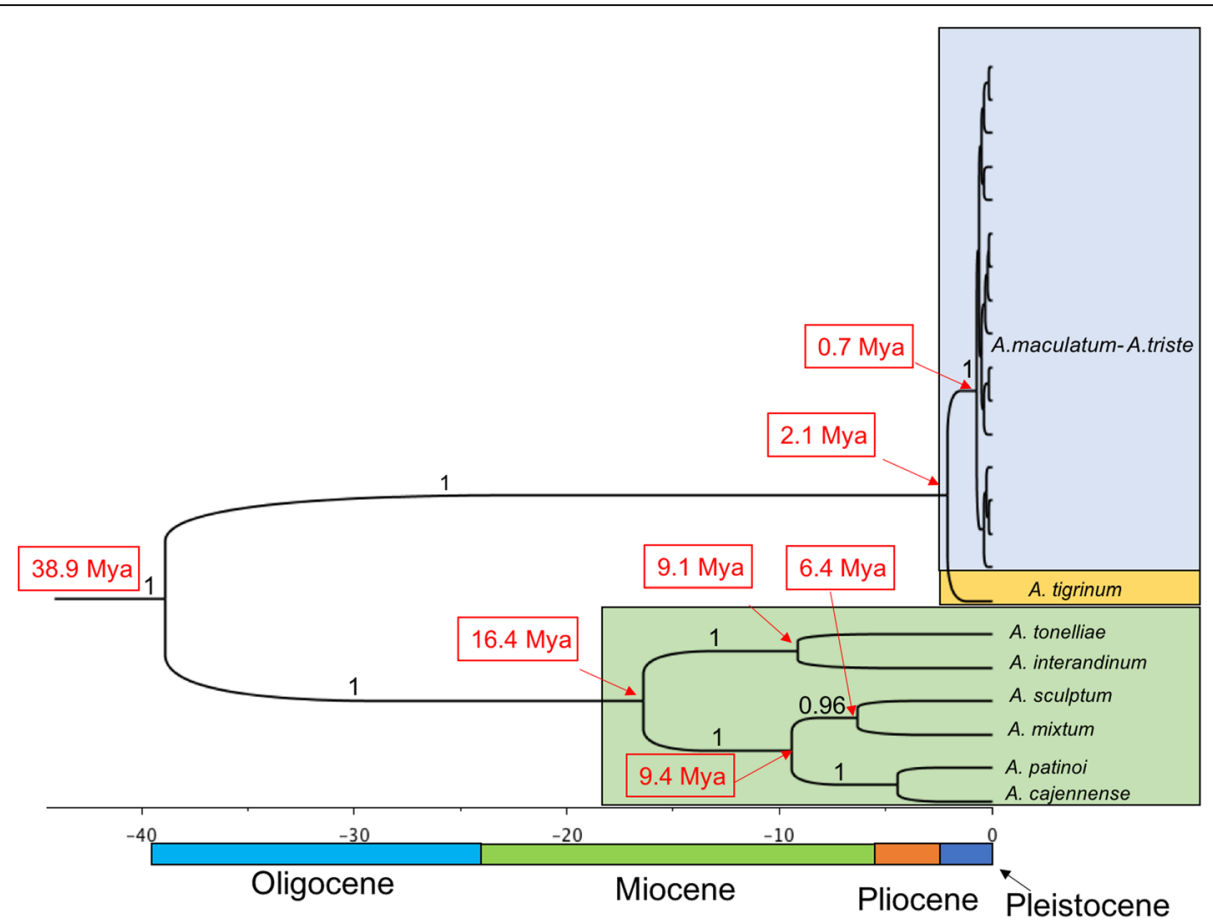

Fig. 8 Tentative node dating of the main nodes on a tree generated by BEAST using the relaxed clock method. Numbers (in black) over branches represent posterior probability support, while the dates are represented in red and in millions of years ago (Mya)

of a strict molecular clock in BEAST, we selected the relaxed molecular clock option which allows for variable mutation rates because mutation rates differed significantly between ingroup and outgroup $(P \leq 0.01)$. The tree generated by BEAST supported the basal subdivision between A. tigrinum and A. maculatum - A. triste, but did not resolve relationships within the $A$. maculatum - A. triste polytomic clade (Fig. 8). By using a single calibration point, we first confirmed that the nodes within the $A$. cajennense group were consistent with prior findings [36] associating nodes with known biogeographical vicariant events. The diversification of the $A$. maculatum group of taxa appeared to be more recent with the split between A. tigrinum and the A. maculatum - A. triste clades dating back to approximately 2.1 Mya (95\% confidence interval: $0.9-3.3 \mathrm{Mya}$ ) and the $A$. maculatum - A. triste radiation beginning no sooner than 720,000 years ago (95\% confidence interval: $0.3-1.2$ Mya) in the mid-Pleistocene (Fig. 8). The split between the outgroup and the ingroup dated was estimated at 39 Mya (95\% confidence interval: 14-70 Mya).

\section{Discussion}

Morphological reassessment of the A. maculatum - A. triste specimens revealed four morphotypes distinguishable by four distinct sets of characters. Morphotype I includes specimens belonging to populations from Argentina, Paraguay, Uruguay and southern Brazil.
These ticks were assigned to the taxon A. triste (s.s.) because their morphology is compatible with the morphology of the type-specimens of A. triste (ZMB 1046) from Montevideo, Uruguay (see [4]). Morphotype II includes specimens from eastern USA, Colombia, Guatemala, Honduras, Mexico and Venezuela, with a morphology matching the type-specimens of $A$. maculatum (s.s.) (ZMB 1044) also described by Koch [4] from a type-locality reported as "Carolina", USA (possibly in one of the Carolina States which are included in the present distribution of $A$. maculatum). Morphotypes I and II have so far been differentiated by examining the tibial armature of legs II-IV, the shape of the male spiracular plates and, in some cases, scutal ornamentation in females. The remaining two morphotypes present a combination of characters that precludes a strict assignation to either A. triste or A. maculatum, which explain why Peruvian and Chilean samples have alternatively been considered to be $A$. maculatum or A triste [10, 37, 38], and Arizona samples have been assigned to A. triste [11], based on tibial armature morphology. Morphotype III includes ticks from the southwestern USA (Texas and Arizona) and northern Mexico. It could also correspond to what Guzman-Cornejo et al. [22] described as A. triste from Mexico, although the specimens should be reexamined. They have one spine and one seta on tibiae of legs II-IV, comma-shaped spiracular plates (elongated in males), and the scutum of females with a central spot 
Table 2 Summary of distinctive morphological character combinations in the four different morphotypes

\begin{tabular}{|c|c|c|c|c|c|c|c|}
\hline \multirow[t]{2}{*}{ Morphotype } & \multirow[t]{2}{*}{ Origin } & \multirow{2}{*}{$\begin{array}{l}\text { Males } \\
\text { Scutes } \\
\text { on ventral } \\
\text { festoons }\end{array}$} & \multirow{2}{*}{$\begin{array}{l}\text { Males } \\
\text { Postero-median } \\
\text { spot in relation } \\
\text { to width of } \\
\text { adjacent stripe }\end{array}$} & \multirow{2}{*}{$\begin{array}{l}\text { Males + Females } \\
\text { Tibial armature }\end{array}$} & \multirow{2}{*}{$\begin{array}{l}\text { Males } \\
\text { Spiracular plates } \\
\text { (width of projection } \\
\text { in relation to width } \\
\text { of adjacent festoon) }\end{array}$} & \multirow{2}{*}{$\begin{array}{l}\text { Females } \\
\text { Spiracular plates } \\
\text { (width of projection } \\
\text { in relation to width } \\
\text { of adjacent festoon) }\end{array}$} & \multirow{2}{*}{$\begin{array}{l}\text { Females } \\
\text { Brown central } \\
\text { spot }\end{array}$} \\
\hline & & & & & & & \\
\hline I & $U Y, A R, B R$ & Present & Wider & $\begin{array}{l}1 \text { spine }+1 \text { stout } \\
\text { seta }\end{array}$ & $\begin{array}{l}\text { Oval, with short wide } \\
\text { projection ( } \pm 1 \text { festoon) }\end{array}$ & $\begin{array}{l}\text { Comma-shaped, wide } \\
( \pm 1 \text { festoon) }\end{array}$ & $\begin{array}{l}\text { Reaching posterior } \\
\text { margin of scutum }\end{array}$ \\
\hline$\|$ & $\begin{array}{l}\text { USA, CO, } \\
\text { CR, VE }\end{array}$ & $\begin{array}{l}\text { Present, less } \\
\text { conspicuous }\end{array}$ & Wider & $\begin{array}{l}2 \text { spines of almost } \\
\text { equal length }\end{array}$ & $\begin{array}{l}\text { Comma-shaped with } \\
\text { narrow projection } \\
\text { ( } \pm 1 / 2 \text { festoon) }\end{array}$ & $\begin{array}{l}\text { Comma-shaped, with } \\
\text { narrow projection } \\
( \pm 1 / 2 \text { festoon) }\end{array}$ & $\begin{array}{l}\text { "Mostly" reaching } \\
\text { posterior margin } \\
\text { of scutum }\end{array}$ \\
\hline III & $\begin{array}{l}\text { USA (AZ), } \\
M X\end{array}$ & Present & "Mostly" wider & $\begin{array}{l}1 \text { spine }+1 \text { fine } \\
\text { seta }\end{array}$ & $\begin{array}{l}\text { Comma-shaped, } \\
\text { elongated, with } \\
\text { narrow projection } \\
( \pm 1 / 2 \text { festoon) }\end{array}$ & $\begin{array}{l}\text { Comma-shaped, with } \\
\text { narrow projection } \\
( \pm 1 / 3 \text { festoon) }\end{array}$ & $\begin{array}{l}\text { Reaching posterior } \\
\text { margin of scutum }\end{array}$ \\
\hline IV & $\mathrm{PE}, \mathrm{CH}, \mathrm{EC}$ & Present & Wider & $\begin{array}{l}2 \text { spines of unequal } \\
\text { length }\end{array}$ & $\begin{array}{l}\text { Comma-shaped, } \\
\text { elongated, with } \\
\text { narrow projection } \\
( \pm 1 / 2 \text { festoon) }\end{array}$ & $\begin{array}{l}\text { Comma-shaped, with } \\
\text { narrow projection } \\
( \pm 1 / 2 \text { festoon) }\end{array}$ & $\begin{array}{l}\text { "Mostly" reaching } \\
\text { posterior margin } \\
\text { of scutum }\end{array}$ \\
\hline
\end{tabular}

Abbreviations: AR, Argentina; BR, Brazil; CO, Colombia; CR, Costa Rica; VE, Venezuela; USA, United States; AZ, Arizona; MX, Mexico; PE, Perú; CH, Chile; EC, Ecuador

reaching the posterior margin of the scutum. Morphotypes III and IV are similar, but Morphotype IV is characterized by the presence of two spines which appear to be of different length on tibiae of legs II-IV. However, when these phenotypic differences are closely scrutinized, with the exceptions of the overall shape of the spiracular plates and differences in thickness of the tibial setae on legs II-IV, none of the other phenotypic features appear to be fixed when sufficient numbers of ticks from each area are observed. All other differentiating features are shared by at least two of the four morphotypes (Table 2). This situation raises the question of whether we are dealing with four different species, less than four, or a single species.

Analyses of the individual gene sequences, commonly used to reassess taxonomic issues within Amblyomma taxa [36, 39-41], were unable to resolve relationships within the group. Only analyses of the concatenated datasets could subdivide the A. maculatum - A. triste group into three supported clades characterized by distinct geographical ranges, but not always by distinct morphologies since Morphotype III is not monophyletic but is instead a basal polytomy within the Morphotype II+III cluster. The genetic similarity, often identity, particularly between mitochondrial sequences from eastern USA and Arizona-Mexico (Additional files 1, 2, 3, 4: Tables S1-S4) indicate that the spurs and the features of the spiracular plate commonly used to distinguish $A$. maculatum from $A$. triste are not species-specific characters. Molecular divergence values between morphotypes II and III are equivalent to diversity within Morphotype I, further indicating that the two morphologically distinct but genetically uniform North American populations, can be considered to belong to the same species, $A$. maculatum, which extends into parts of northern South America. Although we only obtained sequences from a single Colombian sample of $\mathrm{A}$. maculatum, it consistently clustered with a sample from Georgia, USA (21D) as a slightly separated lineage within the Morphotype II+III clade, confirming the occurrence of the species in Colombia, as recently shown in a study on the diversity of Colombian ticks [21].

If the morphology of the tibial armature and of the spiracular plates cannot consistently be associated with speciation, the next question is whether we should consider Morphotype I, i.e. A. triste (s.s.), and Morphotype IV to be different species from $A$. maculatum. Genetic distance between populations of ticks that are geographically remote or even disjunct in their distribution is to be expected. Nevertheless, even the largest distances within the A.maculatum - A. triste group are comparable to intraspecific distances in other Amblyomma [36, 39, 40] and Ixodes [42-44] species when corresponding gene distances are compared separately. Nevertheless, mutation rates can vary between more or less related lineages and comparisons of simple distance values between distant taxa can be misleading. In this case, however, we have verified that within the $A$. maculatum group mutation rates are not significantly different between the sister lineages. Because distance values within Morphotype I (0.91-1.22\%) are equal to or higher than distances between the same Morphotypes I and II+III (0.32-0.71\%), we deduce that $A$. triste should also be returned to a junior synonym of $A$. maculatum (priority by page number in [4]). Although distances between Morphotype IV and the other morphotypes are slightly higher (1.22-1.70\%), they are significantly lower than those between $A$. tigrinum and all morphotypes (3.91-4.31\%).

In this study we are facing a situation in which morphological and molecular data are not offering a congruent solution, in a taxon (the ingroup) that radiated very 
recently. Our data do not support consistently the subdivision of this group into four species, although we agree that some of the presented evidence would provide justification. For now, we can only base our conclusion on our results, but there is no doubt that additional studies at a finer taxonomic level based either on nuclear codominant very variable markers (microsatellites or SNPs) and cross-breeding experiments will help in reaching more satisfying conclusions.

In the case of $A$. tigrinum, this species differs from $A$. triste and $A$. maculatum by host preferences [17, 45-47], ecological preferences [17] and morphology of both adults and immature stages [17]. Molecularly, A. tigrinum sequences always clustered in a well-defined monophyletic lineage. Although divergence values between $A$. tigrinum and A. maculatum - A. triste are moderately higher than intraspecific values, they remain much lower than the interspecific distances recorded between outgroup species, and between outgroup and ingroup taxa. More importantly, the variable nuclear gene used in this study (ITS2) which has successfully been used for taxonomic reassessments among South American Amblyomma species [36, 41], like some of the mitochondrial genes, included A. tigrinum in a polytomic ingroup and did not support a clear split between the taxa. For the time being, we do not consider A. tigrinum to be a synonym of A. maculatum, as proposed by Neumann [6] but to be a separate taxon of very recent radiation from $A$. maculatum - A. triste. After all, it has been shown that in some cases differences in host association can trigger rapid genetic divergence in tick species [48]. Only cross-breeding experiments are likely to determine whether the time elapsed since the divergence of the two lineages (2.1 Mya) has been sufficient for them to become different species.

In terms of node dating, the A. maculatum - A. triste radiation initiated no earlier than 700,000 years ago, a time frame not too different from that evaluated for the intraspecific diversification of A. variegatum [39] and of Ixodes scapularis Say [43, 44]. The branch lengths within the A. maculatum - A. triste clade are very short compared to those between the ingroup and outgroup and between different outgroup species (Fig. 8). This indicates that the radiation from the earliest common ancestor of the A. maculatum group was very rapid, almost explosive. If we compare our tree topology to that of other Amblyomma groups (the A. cajennense and A. parvum groups) with similar geographical distributions $[36,40]$, it appears that the A. maculatum group colonized its present range much later and very rapidly. Alternatively, we might consider the possibility that earlier widespread populations went almost extinct and that the present lineages arose through a major bottleneck. It could be argued that Morphotypes II-III-IV have evolved along the same biogeographical patterns described for $A$. mixtum [36] a species which exploited the closing of the Panama Isthmus (3 Mya) for dispersal through a wide area encompassing Colombia, the coast of Ecuador, Central America and southern North America. This could speculatively have been followed by allopatric incipient diversification occurring along the western coast of northern South America (Peru), in the Madrean Archipelago (Sky Islands) north of Mexico and in southern Arizona [49], while the most common morphotype (II) survived as such in Colombia and southeastern North America. Vicariant events cannot, however, explain the split between Morphotype II and I.

Additional studies with different molecular markers and many more samples, could help to better reconstruct the demographic history of the group.

\section{Conclusions}

In summary, the data and evidence presented are inconsistent with the hypothesis that $A$. maculatum, A. triste and $A$. tigrinum represent three separate species. The evidence presented in this study supports the conspecificity of $A$. maculatum and $A$. triste. It is possible that the minor observed morphological differences are the result of a very rapid adaptation to slightly different environments not yet associated with sufficient genetic differentiation to support speciation. Further studies, especially cross-breeding experiments, should follow, as they may add valuable information and further support (or reject) the hypothesis of conspecificity of $A$. maculatum and $A$. triste raised in this study.

\section{Methods}

\section{Morphological analysis}

Morphological analyses were performed through examination of adult ticks deposited in the following tick collections: (i) Tick Collection of the Instituto Nacional de Tecnología Agropecuaria, Estación Experimental Agropecuaria Rafaela, Rafaela, Argentina (INTA); (ii) U.S. National Tick Collection, Georgia Southern University, Statesboro, Georgia, USA (USNTC, RML accession numbers); (iii) Tick Collection of the Departamento de Parasitología Veterinaria, Facultad de Veterinaria, Universidad de la República, Salto, Uruguay (DPVURU); and (iv) Coleçao Nacional de Carrapatos da Faculdade de Medicina Veterinaria e Zootecnia, Universidade de São Paulo, Brazil (CNC). Ticks determined as A. triste, A. maculatum and Amblyomma sp. cf. A. triste - A. maculatum from Argentina, Brazil, Chile, Colombia, Ecuador, Guatemala, Honduras, Mexico, Nicaragua, Peru, Uruguay, USA and Venezuela, were included in the morphological analysis. We also have examined the type-specimens of $A$. triste, A. maculatum and A. tigrinum from the Zoological Museum in Berlin (ZMB). The 
terminology used in the morphological diagnoses follows Nava et al. [17].

\section{Sampling for molecular analyses}

Our sample included a total of 109 adult specimens initially identified as A. maculatum, A. triste or A. tigrinum, and two specimens identified as $A$. parvitarsum and $A$. neumanni. Amblyomma maculatum ticks were from the USA (Georgia and Florida), Peru and Colombia; $A$. triste were from Argentina, Brazil, Uruguay, Peru, Mexico and Arizona (USA); A. tigrinum were from Argentina and Brazil; and $A$. parvitarsum and A. neumanni were from Argentina. When available, specimens from several localities were included to consider variation between and within different regions (Table 1). The ticks were subsequently reclassified by using a newly outlined phenotypic subdivision (see Results section) of $A$. maculatum - A. triste into four morphotypes (Table 1). Ticks were obtained from 28 localities and 7 countries (Fig. 1), and coded as follows: Argentina (BA, Buenos Aires; CR, Corrientes; FO, Formosa; SDE, Santiago del Estero), Brazil (GO, Goiás; MGS, Mato Grosso do Sul; SP, Sao Paulo), Colombia (SR, Santander), Peru (PU) (IC, Ica; TU, Tumbes; PI, Piura), Mexico (MX) (HO, Sonora), and the USA (FL, Florida; GA, Georgia; AZ, Arizona) (Table 1).

\section{DNA extraction, PCR and sequencing}

Tick DNA was extracted and, when possible, the exoskeletons were preserved for further morphological analysis following previously published protocols $[39,50]$. A small portion of the postero-lateral idiosoma of each tick was removed by using a sterile disposable scalpel and the tick was incubated overnight in $180 \mu \mathrm{l}$ Qiagen ATL lysis buffer (Qiagen, Valencia, CA, USA) and $40 \mu \mathrm{l}$ of a $14.3 \mathrm{mg} / \mathrm{ml}$ solution of proteinase K (Roche Applied Sciences, Indianapolis, IN, USA). After repeated vortexing and ascertaining that the lysis was complete, each exoskeleton was stored in $70 \%$ ethanol and kept as a voucher specimen. The lysed tissues were further processed as previously described $[36,39,50]$. Five mitochondrial gene sequences, $12 S$ rDNA (small subunit ribosomal RNA), $16 S$ rDNA (small subunit ribosomal RNA), cox1 (cythochrome $c$ oxidase subunit 1), the control region or d-loop (DL) were amplified with previously reported sets of primers [36, 39, 50-52]. In addition, a portion of the nuclear ribosomal internal transcribed spacer 2 (ITS2) was amplified by slightly modifying a previously published protocol to include 35 instead of 27 annealing cycles $[39,53]$. PCRs were performed using a MasterTaq kit (5-Prime, Gaithersburg, MD, USA). Each reaction contained $2.5 \mu \mathrm{l}$ of tick DNA, $2.5 \mu \mathrm{l}$ of $10 \times$ Taq buffer, 5 $\mu \mathrm{l}$ of $5 \times$ TaqMaster PCR Enhancer, $1.5 \mu \mathrm{l}$ of MgAc (25 $\mathrm{mM}), 0.5 \mu \mathrm{l}$ of dNTP mix (10 mM each), $0.1 \mu \mathrm{l}$ of Taq polymerase $(5 \mathrm{U} / \mu \mathrm{l}), 1.25 \mu \mathrm{l}$ of each primer from a 10 $\mathrm{pmol} / \mu \mathrm{l}$ stock solution (Invitrogen, Life Technologies Corporation, Grand Island, NY, USA) and $14.6 \mu \mathrm{l}$ of molecular biology grade water. The two DNA strands of each amplicon were purified and sequenced at the High-Throughput Genomics Unit (HTGU, University of Washington, Seattle, WA, USA or at Eurofins, Louisville, KY, USA) and were assembled with Sequencer 4.5 (Gene Codes Corporation, Ann Arbor, MI, USA).

\section{Phylogenetic analyses}

Sequences were manually aligned with McClade 4.07 OSX (Sinauer Associates, Sunderland, MA, USA) [54]. Secondary structure was considered in aligning ribosomal genes [50] and DL [55]. Codon organization was taken into account when aligning the $\operatorname{cox} 1$ data set. Each data set was analyzed by maximum parsimony (MP) with PAUP [56] and by Bayesian inference analysis (BI) using MrBayes 3.2.4 [57, 58]. Branch support was assessed by bootstrap analysis (1000 replicates) with PAUP for MP, and by posterior probability with MrBayes for BI. MP heuristic searches were performed by branch-swapping using the tree bisection-reconnection (TBR) algorithm. Maximum likelihood distances were calculated after the nucleotide substitution model best fitting the data was selected by JModeltest v.2.1.7 [59, 60]. Maximum likelihood pairwise distances were calculated based in the selected model by using PAUP. Two runs with four chains each were run simultaneously for BI analyses (1,000,000 generations). Trees were sampled every 100 iterations. Trees saved before the average standard deviation of split fragments converged to a value $<0.01$ were discarded from the final sample. When necessary, the number of generations was increased so that the number of discarded samples would not exceed $25 \%$ of the total sampled trees. The $50 \%$ majority-rule consensus tree of the remaining trees was inferred, and posterior probabilities recorded for each branch. Two concatenated datasets, one (mtDNA) including 4 mitochondrial genes (12S rDNA, $16 S$ rDNA, DL and cox 1 ) and one including the same 4 gene sequences and ITS2 sequences $(\mathrm{mt}+\mathrm{nDNA})$, were analyzed following the same procedure outlined for the separate analyses. $A$. parvitarsum and $A$. neumanni were used as outgroups in our analyses because they are recognized as being close relatives of our ingroup taxa [3]. Additional species were considered as possible outgroups and preliminary analyses were performed with the following taxa: $A$. aureolatum; Amblyomma coelebs Neumann, 1899; Amblyomma dubitatum Neumann, 1899; Amblyomma oblongoguttatum Koch, 1844; and A. ovale. These alternative outgroups were discarded because they were too distantly related to the ingroup to provide resolution within ingroup taxa. 


\section{Molecular clock and divergence dates}

Additional outgroup sequences available for the 6 species of Amblyomma species now included in the A. cajennense complex [Amblyomma cajennense (Fabricius, 1787), Amblyomma sculptum (Berlese, 1888), Amblyomma mixtum (Koch, 1844), Amblyomma tonelliae (Nava, Beati \& Labruna, 2014), Amblyomma patinoi (Labruna, Nava \& Beati, 2014), and Amblyomma interandinum (Beati, Nava \& Cáceres, 2014)] were incorporated in a concatenated dataset (12S rDNA, DL and ITS2), including the unique A. maculatum, $A$. triste and $A$. tigrinum sequences. Amblyomma cajennense has been shown to be sufficiently close to the $A$. maculatum group to serve as alternate outgroup [3], with the additional advantage of having already been analyzed in terms of phylogeographical evolution and node dating [36]. In order to test substitution rate variation among lineages, relative-rate tests were applied to the main sister clades (A. tigrinum vs A. maculatum A. triste, Peruvian vs all other clades, southern South American vs North American) by using DAMBE [61]. In addition, the molecular clock hypothesis was tested by the least square method and the likelihood ratio-test also in DAMBE [61]. A tentative estimate of the divergence time for the A. maculatum group of species was performed by using the relaxed molecular clock model implemented in BEAST v.1.7.4 [62, 63]. Monophyly was constrained for clades supported in the phylogenetic analysis. The tree prior was set to Calibrated Yule Process, and the molecular clock set to uncorrelated lognormal distribution. Chain lengths were set to $10,000,000$ and data were sampled every 1000 iterations with a random starting tree. After deleting $5 \%$ of the generated trees, the remaining trees were summarized in a combined $50 \%$ maximum clade credibility tree by using TreeAnnotator v.1.7.4. Data were analyzed with Tracer v.1.6.0 and FigTree v.1.3.1 was used to visualize tree structure, with mean divergence times. Node calibration was based on previously generated estimates for the A. cajennense complex [36, 64], with the node at the origin of the $A$. cajennense radiation set at 17 \pm 1.5 Mya.

\section{Additional files}

Additional file 1: Table S1. Geographical distribution of the 125 rDNA haplotypes. Abbreviations: PI, Piura; TU, Tumbes; GA, Georgia; FL, Florida; AZ, Arizona; SDE, Santiago del Estero; BA, Buenos Aires; CR, Corrientes; FO, Formosa; MGS, Mato Grosso do Sul; SP, São Paulo; GO, Goias; \$, A. maculatum; \%, A. triste; \#, A. triste - A. maculatum; * A. tigrinum. (XLSX $11 \mathrm{~kb}$ )

Additional file 2: Table S2. Geographical distribution of the 165 rDNA haplotypes. Abbreviations: PI, Piura; TU, Tumbes; IC, Ica; GA, Georgia; FL, Florida; AZ, Arizona; SDE, Santiago del Estero; BA, Buenos Aires; CR, Corrientes; FO, Formosa; MGS, Mato Grosso do Sul; SP, São Paulo; GO, Goias; \$, A. maculatum; \%, A. triste; \#, A. triste - A. maculatum; *, A. tigrinum. (XLSX $13 \mathrm{~kb})$

Additional file 3: Table S3. Geographical distribution of the cox1 haplotypes. Abbreviations: PI, Piura; TU, Tumbes; IC, Ica; GA, Georgia; FL,
Florida; AZ, Arizona; SDE, Santiago del Estero; BA, Buenos Aires; CR, Corrientes; FO, Formosa; MGS, Mato Grosso do Sul; SP, São Paulo; GO, Goias; \$, A. maculatum; \%, A. triste; \#, A. triste - A. maculatum; *, A. tigrinum. (XLSX $13 \mathrm{~kb}$ )

Additional file 4: Table S4. Geographical distribution of the DL haplotypes. Abbreviations: PI, Piura; TU, Tumbes; IC, Ica; GA, Georgia; FL, Florida; AZ, Arizona; SDE, Santiago del Estero; BA, Buenos Aires; CR, Corrientes; FO, Formosa; MGS, Mato Grosso do Sul; SP, São Paulo; GO, Goias; \$, A. maculatum; \%, A. triste; \#, A. triste - A. maculatum; *, A. tigrinum. (XLSX $12 \mathrm{~kb}$ )

Additional file 5: Table S5. Geographical distribution of the ITS2 genotypes. Abbreviations: PI, Piura; TU, Tumbes; GA, Georgia; FL, Florida; AZ, Arizona; BA, Buenos Aires; CR, Corrientes; FO, Formosa; MGS, Mato Grosso do Sul; SP, São Paulo; GO, Goias; SDE, Santiago del Estero; \$, A. maculatum; \%, A. triste; \#, A.triste - A. maculatum; * A. tigrinum. (XLSX $10 \mathrm{~kb}$ )

\section{Abbreviations}

BI: Bayesian inference; MP: Maximum parsimony; mtDNA: Mitochondrial DNA; Mya: Millions of years ago; nDNA: Nuclear DNA

\section{Acknowledgements}

The authors would like to thank two anonymous reviewers who made insightful comments and useful suggestions which helped improving the manuscript.

\section{Funding}

This work was supported by the National Science Foundation, United States (grant number 1026146) to LB.

\section{Availability of data and materials}

The datasets generated during the present study are available in the GenBank repository. The accession numbers are in this published article (Results section)

\section{Authors' contributions}

$L B, S N$ and $P L$ designed the study, analyzed the data and drafted the manuscript. PL, SN, LMU, AGC, JDM, JLE, DDM, MBL, LAD, MEJA, CDP, MPJS, JMV, AAG and LB made contributions to the acquisition and interpretation of data. All authors read and approved the final manuscript.

\section{Ethics approval and consent to participate}

Not applicable

\section{Consent for publication \\ Not applicable.}

\section{Competing interests}

The authors declare that they have no competing interests.

\section{Publisher's Note}

Springer Nature remains neutral with regard to jurisdictional claims in published maps and institutional affiliations.

\section{Author details}

'U. S. National Tick Collection, Institute for Coastal Plain Science, Georgia Southern University, Statesboro, GA 30460, USA. ${ }^{2}$ Present address: Department of Evolution, Ecology, and Organismal Biology, The Ohio State University, Columbus, OH 43212, USA. ${ }^{3}$ Instituto Nacional de Tecnologia Agropecuaria, Estación Experimental Agropecuaria Rafaela, Santa Fe, Argentina. ${ }^{4}$ Laboratorio de Entomología, Instituto Nacional de Salud, Lima, Peru. ${ }^{5}$ Departamento Académico de Microbiologia Médica, Facultad de Medicina, Universidad Nacional Mayor de San Marcos, Lima, Peru.

'Laboratorio de Entomología, Instituto Nacional de Salud, Lima, Peru. ${ }^{7}$ Department of Medicine and Health Sciences, University of Sonora, Sonora, Mexico. ${ }^{8}$ Department of Agronomic and Veterinary Sciences, Technologic Institute of Sonora, Ciudad Obregón, Sonora, Mexico. ${ }^{9}$ Departamento de Medicina Veterinária Preventiva e Saúde Animal, Faculdade de Medicina Veterinária e Zootecnia, Universidade de São Paulo, São Paulo, Brazil.

${ }^{10}$ Department of Biology, Georgia Southern University, Statesboro, GA 30460, 
USA. ${ }^{11}$ Rickettsial Zoonoses Branch, National Center for Emerging and Zoonotic Infectious Diseases, Centers for Disease Control and Prevention, Atlanta, GA, USA. ${ }^{12}$ Laboratório de Ixodologia, Faculdade de Medicina Veterinária, Universidade Federal de Uberlândia, Uberlândia, Minas Gerais, Brazil. ${ }^{13}$ Departamento de Parasitología Veterinaria, Facultad de Veterinaria, Universidad de la República, Regional Norte - Salto, Rivera 1350, 50000 Salto, $\mathrm{CP}$, Uruguay.

\section{Received: 26 June 2018 Accepted: 6 November 2018} Published online: 29 November 2018

\section{References}

1. Camicas JL, Hervy JP, Adam F, Morel PC. Les tiques du monde. Nomenclature, stades décrits, hôtes, répartition (Acarida, Ixodida). Paris: ORSTOM; 1998.

2. Santos Dias JAT. Contribuição para o estudo da sistemática dos ácaros da suborden Ixodoidea Banks, 1894. Mém Museu Zool Univ Coimbra. 1963; 285:34.

3. Estrada-Peña A, Venzal JM, Mangold AJ, Cafrune MM, Guglielmone AA. The Amblyomma maculatum Koch, 1844 (Acari: Ixodidae: Amblyomminae) tick group: diagnostic characters, description of the larva of A. parvitarsum Neumann, 1901, $16 S$ rDNA sequences, distribution and hosts. Syst Parasitol. 2005;60:99-112.

4. Koch CL. Systematische übersicht über die Ordnung der Zecken. Arch Naturgesch. 1844;10:217-39.

5. Koch CL. Übersicht des Arachnidensystems, vol. IV. Nurnberg: J.L. Lotzbeck; 1847. p. 136

6. Neumann LG. Révision de la famille des Ixodides. Mem Soc Zool de France. 1899;12:107-294

7. Robinson LE. Ticks: A Monograph of the Ixodoidea. Part IV. The Genus Amblyomma. Cambridge: Cambridge University Press; 1926.

8. Kohls GM. Concerning the identity of Amblyomma maculatum, A. tigrinum, A. triste and A. ovatum of Koch, 1844. Proc Entomol Soc Wash. 1956;58:143-7.

9. Guglielmone AA, Nava S, Mastropaolo M, Mangold AJ. Distribution and genetic variation of Amblyomma triste (Acari: Ixodidae) in Argentina. Ticks Tick Borne Dis. 2013;4:386-90.

10. Mendoza Uribe L, Chavez Chorocco J. Ampliacion geografica de siete especies de Amblyomma (Acari: Ixodidae) y primer reporte de $A$. oblongoguttatum Koch, 1844 para Peru. Rev Per Entomol. 2004;44:69-72.

11. Mertins JW, Moorhouse AS, Alfred JT, Hutcheson HJ. Amblyomma triste (Acari: Ixodidae): new North American collection records, including the first from the United States. J Med Entomol. 2010;47:536-42.

12. Aragão $\mathrm{H}$, da Fonseca F. Notas de ixodología. VIII. Lista e chave para os representantes da fauna ixodológica brasileira. Mem Inst Oswaldo Cruz. 1961;59:115-29.

13. Tagle I, Alvarez V. Existencia de Amblyomma maculatum Koch, 1844 en zorros de Chile. Bol Chileno Parasitol. 1957;12:66.

14. Tagle I, Alvarez V. Rectificacion de diagnostico: Amblyomma tigrinum, Koch, 1844 en lugar de Amblyomma maculatum Koch. Bol Chileno Parasitol. 1959; 3:56-7.

15. Estrada-Peña A, Venzal JM, Guglielmone AA. Amblyomma dubitatum Neumann: description of nymph and redescription of adults, together with the description of the immature stages of A. triste Koch. Acarologia. 2002:42:323-33.

16. Martins TF, Labruna MB, Mangold AJ, Cafrune MM, Guglielmone AA, Nava S. Taxonomic key to nymphs of the genus Amblyomma (Acari: Ixodidae) in Argentina, with description and redescription of the nymphal stage of four Amblyomma species. Ticks Tick Borne Dis. 2014;5:753-70.

17. Nava S, Venzal JM, Gonzalez-Acuña D, Martins TF, Guglielmone AA. Ticks of the Southern Cone of America: Diagnosis, Distribution, and Hosts with Taxonomy, Ecology and Sanitary Importance. London: Academic Press; 2017.

18. Guglielmone AA, Mangold AJ, Hadani A. Amblyomma tigrinum Koch, 1844 en la Argentina. Gac Vet B Aires. 1982;46:57-63.

19. Guglielmone AA, Estrada-Peña A, Keirans JE, Robbins RG. Ticks (Acari: Ixodida) of the Neotropical zoogeographic region. Special Publication of the International Consortium on Ticks and Tick-Borne Diseases - 2. Houten, The Netherlands: Atalanta; 2003

20. Jones EK, Clifford CM, Keirans JE, Kohls GM. The ticks of Venezuela (Acarina: Ixodoidea) with a key to the species of Amblyomma in the Western Hemisphere. Brigham Young Univ Science Bull. Biol. Ser. 1972; $17: 1-40$.
21. Rivera-Páez FA, Labruna MB, Martins TF, Perez JE, Castaño-Villa GJ, OssaLópez PA, et al. Contribution to the knowledge of hard ticks (Acari: Ixodidae) in Colombia. Ticks Tick Borne Dis. 2018;9:57-66.

22. Guzmán-Cornejo C, Perez TM, Nava S, Guglielmone AA. Confirmation of the presence of Amblyomma triste Koch, 1844 (Acari: Ixodidae) in Mexico. Syst Appl Acarol. 2006;11:47-50.

23. Allerdice ME, Beati L, Yaglom H, Lash RR. Delgado-De la Mora J, Licona Enriquez JD, et al. Rickettsia parkeri (Rickettsiales: Rickettsiaceae) detected in ticks of the Amblyomma maculatum (Acari: Ixodidae) group collected from multiple locations in southern Arizona. J Med Entomol. 2017;54:1743-9.

24. Ferrari FAG, Goddard J, Paddock CD, Varela-Stokes AS. Rickettsia parkeri and Candidatus Rickettsia andeanae in Gulf Coast ticks, Mississippi, USA. Emerg Inf Dis. 2012:18:1705-7.

25. Herrick KL, Pena SA, Yaglom HD, Layton BJ, Moors A, Loftis AD, et al. Rickettsia parkeri rickettsiosis, Arizona, USA. Emerging Infect Dis. 2016;22:780-5.

26. Lado P, Costa FB, Verdes JM, Labruna MB, Venzal JM. First molecular detection of Rickettsia parkeri in Amblyomma tigrinum and Amblyomma dubitatum ticks from Uruguay. Ticks Tick Borne Dis. 2014;5:660-2.

27. Lado P, Costa FB, Verdes JM, Labruna MB, Venzal JM. Seroepidemiological survey of Rickettsia spp. in dogs from the endemic area of Rickettsia parkeri rickettsiosis in Uruguay. Acta Trop. 2015;146:7-10.

28. Nava S, Elshenawy Y, Eremeeva ME, Sumner JW, Mastropaolo M, Paddock CD. Rickettsia parkeri in Argentina. Emerg Infect Dis. 2008;14:1894-7.

29. Paddock CD, Sumner JW, Comer JA, Zaki SR, Goldsmith CS, Goddard J, et al. Rickettsia parkeri: a newly recognized cause of spotted fever rickettsiosis in the United States. Clin Infect Dis. 2004;38:805-11.

30. Paddock CD, Fournier PE, Sumner JW, Goddard J, Elshenawy Y, Metcalfe MG, et al. Isolation of Rickettsia parkeri and identification of a novel spotted fever group Rickettsia sp. from Gulf Coast Ticks (Amblyomma maculatum) in the United States. Appl Env Microbiol. 2010;76:2689-96.

31. Paddock CD, Goddard J. The evolving medical and veterinary importance of the Gulf Coast tick (Acari: Ixodidae). J Med Entomol. 2015:52:230-52.

32. Romer Y, Seijo AC, Crudo F, Nicholson WL, Varela-Stokes A, Lash RR, Paddock CD. Rickettsia parkeri rickettsiosis Argentina. Emerg Infect Dis. 2011; 17:1169-73

33. Romer Y, Nava S, Govedic F, Cicuttin G, Denison AM, Singleton J, et al. Rickettsia parkeri rickettsiosis in different ecological regions of Argentina and its association with Amblyomma tigrinum as a potential vector. Am J Trop Med Hyg. 2014;91:1156-60.

34. Venzal JM, Portillo A, Estrada-Peña A, Castro O, Cabrera PA, Oteo JA. Rickettsia parkeri in Amblyomma triste from Uruguay. Emerg Inf Dis. 2004;10:1493-5.

35. Ewing SA, Panciera RJ. American canine hepatozoonosis. Clin Microbiol Rev. 2003;16:688-97.

36. Beati L, Nava S, Burkman EJ, Barros-Battesti D, Labruna MB, Guglielmone AA, et al. Amblyomma cajennense (Fabricius, 1787) (Acari: Ixodidae), the Cayenne tick: phylogeography and evidence for allopatric speciation. BMC Evol Biol. 2013:13:267.

37. Abarça K, López J, Acosta-Jamett G, Lepe P, Soares JF. Labruna MB. A third Amblyomma species and the first tick-borne Rickettsia in Chile. J Med Entomol. 2012;49:219-22.

38. Need JT, Dale WE, Keirans JE, Dasch GA. Annotated list of tiks (Acari: Ixodidae, Argasidae) reported in Perú: distribution, hosts, and bibliography. J Med Entomol. 1991;28:590-7.

39. Beati L, Patel J, Lucas-Williams H, Adakal H, Kanduma EG, Tembo-Mwase E, et al. Phylogeography and demographic history of Amblyomma variegatum (Fabricius) (Acari: Ixodidae), the tropical bont tick. Vector Borne Zoonotic Dis. 2012;12:514-25.

40. Lado P, Nava S, Labruna MB, Szabó MPJ, Durden LA, Bermudez S, et al. Amblyomma parvum Aragão, 1908 (Acari: Ixodidae): phylogeography and systematic considerations. Ticks Tick Borne Dis. 2016;7:817-27.

41. Marrelli MT, Souza LF, Marques RC, Labruna MB, Matioli SR, Tonon AP, et al. Taxonomic and phylogenetic relationships between neotropical species of ticks from genus Amblyomma (Acari: Ixodidae) inferred from second internal transcribed spacer sequences of rDNA. J Med Entomol. 2007:44:222-8.

42. Norris DE, Klompen JS, Keirans JE, Black WC. Population genetics of Ixodes scapularis (Acari: Ixodidae) based on mitochondrial 165 and 125 genes. J Med Entomol. 1996;33:78-89.

43. Qiu WG, Dykhuizen DE, Acosta MS, Luft BJ. Geographic uniformity of the Lyme disease spirochete (Borrelia burgdorferi) and its shared history with tick vector (Ixodes scapularis) in the northeastern United States. Genetics. 2002: 160:833-49. 
44. Sakamoto JM, Goddard J, Rasgon JL. Population and demographic structure of Ixodes scapularis Say in the eastern United States. PLoS One. 2014;9:e101389.

45. Guglielmone AA, Mangold AJ, Luciani CE, Viñabal A. Amblyomma tigrinum (Acari: Ixodidaae) in relation to phytogeography of central-northern Argentina with notes on hosts and seasonal distribution. Exp Appl Acarol. 2000;24:983-9.

46. Hoogstraal H, Aeschlimann A. Tick-host specificity. Bull Soc Entomol Suisse. 1982;55:5-32.

47. Ivancovich JC. Reclasificacion de algunas especies de garrapatas del genero Amblyomma (Ixodoidea) en la Argentina. Rev Inv Agropec. 1980;15:673-82.

48. De Meeûs T, Koffi BB, Barré N, de Garine- Wichatitsky M, Chevillon C. Swift sympatric adaptation of a species of cattle tick to a new deer host in New Caledonia. Infect Genet Evol. 2010;10:976-83.

49. Moore W, Meyer WM, Eble JA, Franklin K, Wiens JF, Brusca RC. Introduction to the Arizona Sky Island Arthropod Project (ASAP): Systematics, biogeography, ecology, and population genetics of Arthropods of the Madrean Sky Islands. Proc RMRS. 2013;2013:144-68.

50. Beati L, Keirans JE. Analysis of the systematic relationships among ticks of the genera Rhipicephalus and Boophilus (Acari: Ixodidae) based on mitochondrial 125 ribosomal DNA gene sequences and morphological characters. J Parasitol. 2001;87:32-48.

51. Barrett RDH, Hebert PDN. Identifying spiders through DNA barcodes. Can J Zool. 2005;83:481-91.

52. Mangold AJ, Bargues MD, Mas-Coma S. Mitochondrial $16 \mathrm{~S}$ rDNA sequences and phylogenetic relationships of species of Rhipicephalus and other tick genera among Metastriata (Acari: Ixodidae). Parasitol Res. 1998:84:478-84.

53. McLain DK, Wesson DM, Oliver $\mathrm{JH}$, Collins FH. Variation in ribosomal DNA internal transcribed spacers 1 among eastern populations of Ixodes scapularis (Acari: Ixodidae). J Med Entomol. 1995;32:353-60.

54. Maddison DR, Maddison WP. MacClade 4. Analysis of Phylogeny and Character Evolution. Sunderland, MA: Sinauer Associates; 2000.

55. Zhang DX, Hewitt G. Insect mitochondrial control region: a review of its structure, evolution and usefulness in evolutionary studies. Biochem Syst Ecol. 1997;25:99-120

56. Swofford DL. PAUP*: Phylogenetic Analysis Using Parsimony (and other methods) 4.0 Beta. Sunderland, MA: Sinauer Associates; 2000.

57. Huelsenbeck JP, Ronquist F. MRBAYES: Bayesian inference of phylogenetic trees. Bioinformatics. 2001;17:754-5.

58. Ronquist F, Huelsenbeck J, Teslenkoet M. MrBayes version 3.2 Manual: Tutorials and odel summaries, 2011. http://mrbayes.sourceforge.net/manual.php

59. Darriba D, Taboada GL, Doallo R, Posada D. jModelTest 2: more models, new heuristics and parallel computing. Nat Methods. 2012;9:772.

60. Guindon S, Gascuel O. A simple, fast, and accurate algorithm to estimate large phylogenies by maximum likelihood. Syst Biol. 2003;52:696-704.

61. Xia X, Xie Z. DAMBE: software package for data analysis in molecular biology and evolution. J Hered. 2001;92:371-3.

62. Drummond AJ, Ho S, Phillips MJ, Rambaut A. Relaxed phylogenetics and dating with confidence. PLoS Biol. 2006:4:e88.

63. Drummond AJ, Rambaut A. BEAST: Bayesian evolutionary analysis by sampling trees. BMC Evol Biol. 2007;7:214.

64. Nava S, Beati L, Labruna MB, Caceres AG, Mangold AJ, Guglielmone AA. Reassessment of the taxonomic status of Amblyomma cajennense ( Fabricius, 1787) with the description of three new species, Amblyomma tonelliae n. sp., Amblyomma interandinum n. sp. and Amblyomma patinoi n. sp., and reinstatement of Amblyomma mixtum Koch, 1844, and Amblyomma sculptum Berlese, 1888 (Ixodida: Ixodidae). Ticks Tick Borne Dis. 2014;5:252-76.

\section{Ready to submit your research? Choose BMC and benefit from:}

- fast, convenient online submission

- thorough peer review by experienced researchers in your field

- rapid publication on acceptance

- support for research data, including large and complex data types

- gold Open Access which fosters wider collaboration and increased citations

- maximum visibility for your research: over $100 \mathrm{M}$ website views per year

At $\mathrm{BMC}$, research is always in progress.

Learn more biomedcentral.com/submissions 